SLAC-PUB-8770

February 2001

\title{
Hadronic Light-Front Wavefunctions and QCD Phenomenology
}

\author{
Stanley J. Brodsky \\ Stanford Linear Accelerator Center \\ Stanford, California 94309 \\ E-mail: sjbth@slac.stanford.edu
}

Invited lectures presented at the

Fifth International Workshop On Particle Physics Phenomenology

Chi-Pen, Taitung, Taiwan

8-11 November 2000

*Work supported by Department of Energy contract DE-AC03-76SF00515. 


\begin{abstract}
A fundamental goal in QCD is to understand the non-perturbative structure of hadrons at the amplitude level — not just the single-particle flavor, momentum, and helicity distributions of the quark constituents, but also the multi-quark, gluonic, and hidden-color correlations intrinsic to hadronic and nuclear wavefunctions. A natural calculus for describing the bound-state structure of relativistic composite systems in quantum field theory is the light-front Fock expansion which encodes the properties of a hadrons in terms of a set of frame-independent $n$-particle wavefunctions. Light-front quantization in the doubly-transverse light-cone gauge has a number of remarkable advantages, including explicit unitarity, a physical Fock expansion, the absence of ghost degrees of freedom, and the decoupling properties needed to prove factorization theorems in high momentum transfer inclusive and exclusive reactions. A number of applications are discussed in these lectures, including semileptonic $B$ decays, two-photon exclusive reactions, and deeply virtual Compton scattering. The relation of the intrinsic sea to the light-front wavefunctions is discussed. A new type of jet production reaction, "self-resolving diffractive interactions" can provide direct information on the light-front wavefunctions of hadrons in terms of their quark and gluon degrees of freedom as well as the composition of nuclei in terms of their nucleon and mesonic degrees of freedom.
\end{abstract}

\title{
1 Introduction
}

In principle, quantum chromodynamics provides a fundamental description of hadron and nuclear physics in terms of quark and gluon degrees of freedom. QCD has been developed and successfully tested extensively, particularly in inclusive and exclusive processes involving collisions at large momentum transfer where factorization theorems and the smallness of the QCD effective coupling allow perturbative predictions. However, despite its empirical successes, many fundamental questions about QCD have not been resolved. These include a rigorous proof of color confinement, the behavior of the QCD coupling at small momenta, a rigorous description of the structure of hadrons in terms of their quark and gluon degrees of freedom, the problem of asymptotic $n$ ! growth of the perturbation theory (renormalon phenomena), the nature of the pomeron and Reggeons, the nature of shadowing and anti-shadowing in nuclear collisions, the apparent conflict between QCD vacuum structure and the small size of the cosmological constant, and the problems of scale and scheme ambiguities in perturbative QCD expansion.

In these lectures I will focus on one of the central questions in QCD - the nonperturbative description of the proton and other hadrons as composites of confined, 
relativistic quark and gluon quanta. The goal is a frame-independent, quantummechanical representation of hadrons at the amplitude level capable of encoding multiquark, hidden-color and gluon momentum, helicity, and flavor correlations in the form of universal process-independent hadron wavefunctions. For example, the measurement and interpretation of the basic parameters of the electroweak theory and $C P$ violation depends on an understanding of the dynamics and phase structure of $B$ decays at the amplitude level. As I will discuss in these lectures, light-front quantization allows a unifying wavefunction representation of non-perturbative hadron dynamics in QCD. Remarkably, it is now possible to measure the wavefunctions of a relativistic hadron by diffractively dissociating it into jets whose momentum distribution is correlated with the valence quarks' momenta [1, 2, 3, 4. It is also particularly important to understand the shape of the gauge- and process-independent meson and baryon valence-quark distribution amplitudes [5] $\phi_{M}(x, Q)$, and $\phi_{B}\left(x_{i}, Q\right)$. These quantities specify how a hadron shares its longitudinal momentum among its valence quarks; they control virtually all exclusive processes involving a hard scale $Q$, including form factors, Compton scattering and photoproduction at large momentum transfer, as well as the decay of a heavy hadron into specific final states [6, 7].

What do we mean by a hadronic wavefunction, and what are its degrees of freedom? In the chiral effective Lagrangian approximation, a baryon can be represented as a classical soliton solution as in the Skyrme model. On the other hand, bag models, the observed baryon spectroscopy, and magnetic moment phenomenology suggests that baryons are composites of three "constituent" quarks or perhaps a quark bound to an effective spin-0 or spin-1 diquark. The cloudy bag model [8] takes into account the effect of meson-baryon fluctuations such as $n \pi^{+}$and $\Lambda K^{+}$in the proton. This in turn implies the existence of sea quarks with an $\bar{u}(x) \neq \bar{d}(x)$ and $s(x) \neq \bar{s}(x)$ asymmetries in the momentum and spin distributions of the quark sea.

The $n$-particle Schrödinger momentum space wavefunction $\psi_{N}\left(\vec{p}_{i}\right)$ of a composite system is the projection of the exact eigenstate of the equal-time Hamiltonian on the $n$-particle states of the non-interacting Hamiltonian, the Fock basis. It represents the amplitude for finding the constituents with three-momentum $\vec{p}_{i}$, orbital angular momentum, and spin, subject to three-momentum conservation and angular momentum sum rules. The constituents are on their mass shell, $E_{i}=\sqrt{\vec{p}_{i}^{2}+m_{i}^{2}}$ but do not conserve energy $\sum_{i=1}^{n} E_{i}>E=\sqrt{\vec{p}^{2}+M^{2}}$. However, in a relativistic quantum theory, a bound-state cannot be represented as a state with a fixed number of constituents. For example, the existence of gluons which propagate between the valence quarks necessarily implies that the hadron wavefunction must describe states with an arbitrary 
number of gluons. Thus a hadronic wavefunction must describe fluctuations in particle number $n$, as well as momenta and spin. One has to take into account fluctuations in the wavefunction which allow for any number of sea quarks, as long as the total quantum numbers of the constituents are compatible with the overall quantum numbers of the baryon.

Gluon exchange between quarks of different nucleons in a nucleus will leave the nucleons in an exited octet state of $S U(3)_{C}$, thus requiring the existence of "hidden color" configurations in a nuclear wavefunction. What is worse is that the description of the state at a given time $t_{0}$ depends not only on the choice of gauge but also on the Lorentz frame of the observer since two observers will differ on their definition of simultaneity.

A number of non-perturbative formalisms have been developed which in principle could provide the exact spectra and wavefunctions of bound states in relativistic quantum field theory, including QCD. These include Euclidean lattice gauge theory, the covariant Bethe-Salpeter bound state equation, and Hamiltonian methods based on equal-time or light-front quantization. Lattice gauge theory, particularly in the heavy quark approximation, has been an enormously helpful guide to the hadronic and glueball spectrum of QCD; however, progress in evaluating hadron wavefunctions has been so far limited to the evaluation of certain moments of hadronic matrix elements [9].

The Bethe-Salpeter formalism in principle provides a rigorous and systematic, renormalizable, and explicitly covariant treatment of bound states of two interacting fields. There has been recent progress applying model forms of Dyson-Schwinger equations to the Bethe-Salpeter formalism in order to incorporate the effects of dynamical symmetry breaking in QCD [10]. However, the actual interaction kernel in the bound state equation and the evaluation of current matrix elements requires a sum over an infinite number of irreducible graphs; the use of a single kernel (the ladder approximation) breaks gauge invariance, crossing symmetry, and has an improper heavy quark limit. It is also intrinsically difficult to apply the Bethe Salpeter to bound states of three or more constituents.

There has been progress applying equal-time Hamiltonian methods to QCD in Coulomb gauge 111]; however, the renormalization procedure in this formalism is not well-defined and the results are typically frame-dependent. The evaluation of current matrix elements in the equal-time formalism is especially problematic since one must include contributions to the current from vacuum excitations. For similar reasons, the expansion of an eigenstate on the equal-time Fock basis of free quanta is not welldefined. 
In the light-front quantization, one takes the light-cone time variable $t+z / c$ as the evolution parameter instead of ordinary time $t$. (The $\hat{z}$ direction is an arbitrary reference direction.) The method is often called "light-front" quantization rather than "light-cone" quantization since the equation $x^{+}=\tau=0$ defines a hyperplane corresponding to a light-front. The light-front fixes the initial boundary conditions of a composite system as its constituents are intercepted by a light-wave evaluated at a specific value of $x^{+}=t+z / c$. In contrast, determining an atomic wavefunction at a given instant $t=t_{0}$ requires measuring the simultaneous scattering of $Z$ photons on the $Z$ electrons.

An extensive review and guide to the light-front quantization literature can be found in Ref. [12]. I will use here the notation $A^{\mu}=\left(A^{+}, A-, A_{\perp}\right)$, where $A^{ \pm}=A^{0} \pm A^{z}$ and the metric $A \cdot B=\frac{1}{2}\left(A^{+} B^{-}+A^{-} B^{+}\right)-A_{\perp} \cdot B_{\perp}$.

The origins of the light-front quantization method can be traced to Dirac [13] who noted that 7 out of the 10 Poincare' generators, including a Lorentz boost $K_{3}$, are kinematical (interaction-independent) when one quantizes a theory at fixed light-cone time. This in turn leads to the remarkable property that the light-front wavefunctions of a hadron are independent of the hadron's total momentum, whether it is at rest or moving. Thus once one has solved for the light-front wavefunctions, one can compute hadron matrix elements of currents between hadronic states of arbitrary momentum. In contrast, knowing the rest frame wavefunction at equal time, does not determine the moving hadron's wavefunction.

Light-front wavefunctions are related to momentum-space Bethe-Salpeter wavefunctions by integrating over the relative momenta $k^{-}=k^{0}-k^{z}$ since this projects out $x^{+}=0$. The light-front Fock space is the eigenstates of the free light-front Hamiltonian; i.e., it is a Hilbert space of non-interacting quarks and gluons, each of which satisfy $k^{2}=m^{2}$ and $k^{-}=\frac{m^{2}+k_{\perp}^{2}}{k^{+}} \geq 0$. The light-front wavefunctions are the projections of the hadronic eigenstate on the light-front Fock basis, the complete set of color singlet states of the free Hamiltonian. An essentially equivalent approach, pioneered by Weinberg [14, 15], is to evaluate the equal-time theory from the perspective of an observer moving in the negative $\hat{z}$ direction with arbitrarily large momentum $P_{z} \rightarrow-\infty$. The light-cone fraction $x=\frac{k^{+}}{p^{+}}$of a constituent can be identified with the longitudinal momentum $x=\frac{k^{z}}{P^{z}}$ in a hadron moving with large momentum $P^{z}$.

It is convenient to define the invariant light-front Hamiltonian: $H_{L C}^{Q C D}=P^{+} P^{-}-\vec{P}_{\perp}^{2}$ where $P^{ \pm}=P^{0} \pm P^{z}$. The operator $P^{-}=i \frac{d}{d \tau}$ generates light-cone time translations. The $P^{+}$and $\vec{P}_{\perp}$ momentum operators are independent of the interactions, and thus are conserved at all orders. The eigen-spectrum of $H_{L C}^{Q C D}$ in principle gives the 
entire mass squared spectrum of color-singlet hadron states in QCD, together with their respective light-front wavefunctions. For example, the proton state satisfies: $H_{L C}^{Q C D}\left|\Psi_{p}\right\rangle=M_{p}^{2}\left|\Psi_{p}\right\rangle$. The projection of the proton's eigensolution $\left|\Psi_{p}\right\rangle$ on the colorsinglet $B=1, Q=1$ eigenstates $\{|n\rangle\}$ of the free Hamiltonian $H_{L C}^{Q C D}(g=0)$ gives the light-front Fock expansion: 16]

$$
\begin{aligned}
\left|\Psi_{p} ; P^{+}, \vec{P}_{\perp}, \lambda\right\rangle= & \sum_{n \geq 3, \lambda_{i}} \int \Pi_{i=1}^{n} \frac{d^{2} k_{\perp i} d x_{i}}{\sqrt{x_{i}} 16 \pi^{3}} \\
& 16 \pi^{3} \delta\left(1-\sum_{j}^{n} x_{j}\right) \delta^{(2)}\left(\sum_{\ell}^{n} \vec{k}_{\perp \ell}\right) \\
& \left|n ; x_{i} P^{+}, x_{i} \vec{P}_{\perp}+\vec{k}_{\perp i}, \lambda_{i}\right\rangle \psi_{n / p}\left(x_{i}, \vec{k}_{\perp i}, \lambda_{i}\right) .
\end{aligned}
$$

It is especially convenient to develop the light-front formalism in the light-cone gauge $A^{+}=A^{0}+A^{z}=0$. In this gauge the $A^{-}$field becomes a dependent degree of freedom, and it can be eliminated from the gauge theory Hamiltonian, with the addition of a set of specific instantaneous light-cone time interactions. In fact in $Q C D(1+$ 1) theory, this instantaneous interaction provides the confining linear $x^{-}$interaction between quarks. In $3+1$ dimensions, the transverse field $A^{\perp}$ propagates massless spinone gluon quanta with polarization vectors [5] which satisfy both the gauge condition $\epsilon_{\lambda}^{+}=0$ and the Lorentz condition $k \cdot \epsilon=0$. Thus no extra condition on the Hilbert space is required.

The light-front Fock wavefunctions $\psi_{n / H}\left(x_{i}, \vec{k}_{\perp i}, \lambda_{i}\right)$ thus interpolate between the hadron $H$ and its quark and gluon degrees of freedom. The light-cone momentum fractions of the constituents, $x_{i}=k_{i}^{+} / P^{+}$with $\sum_{i=1}^{n} x_{i}=1$, and the transverse momenta $\vec{k}_{\perp i}$ with $\sum_{i=1}^{n} \vec{k}_{\perp i}=\overrightarrow{0}_{\perp}$ appear as the momentum coordinates of the light-front Fock wavefunctions. A crucial feature is the frame-independence of the light-front wavefunctions. The $x_{i}$ and $\vec{k}_{\perp i}$ are relative coordinates independent of the hadron's momentum $P^{\mu}$. The actual physical transverse momenta are $\vec{p}_{\perp i}=x_{i} \vec{P}_{\perp}+\vec{k}_{\perp i}$. The $\lambda_{i}$ label the light-front spin $S^{z}$ projections of the quarks and gluons along the $z$ direction. The physical gluon polarization vectors $\epsilon^{\mu}(k, \lambda= \pm 1)$ are specified in light-cone gauge by the conditions $k \cdot \epsilon=0, \eta \cdot \epsilon=\epsilon^{+}=0$. Each light-front Fock wavefunction satisfies conservation of the $z$ projection of angular momentum: $J^{z}=\sum_{i=1}^{n} S_{i}^{z}+\sum_{j=1}^{n-1} l_{j}^{z}$. The sum over $S_{i}^{z}$ represents the contribution of the intrinsic spins of the $n$ Fock state constituents. The sum over orbital angular momenta $l_{j}^{z}=-\mathrm{i}\left(k_{j}^{1} \frac{\partial}{\partial k_{j}^{2}}-k_{j}^{2} \frac{\partial}{\partial k_{j}^{\Gamma}}\right)$ derives from the $n-1$ relative momenta. This excludes the contribution to the orbital angular momentum due to the motion of the center of mass, which is not an intrinsic property of the hadron [17]. 
The interaction Hamiltonian of QCD in light-cone gauge can be derived by systematically applying the Dirac bracket method to identify the independent fields [18. It contains the usual Dirac interactions between the quarks and gluons, the three-point and four-point gluon non-Abelian interactions plus instantaneous light-front-time gluon exchange and quark exchange contributions

$$
\begin{aligned}
\mathcal{H}_{i n t}= & -g \bar{\psi}^{i} \gamma^{\mu} A_{\mu}{ }^{i j} \psi^{j} \\
& +\frac{g}{2} f^{a b c}\left(\partial_{\mu} A^{a}{ }_{\nu}-\partial_{\nu} A^{a}{ }_{\mu}\right) A^{b \mu} A^{c \nu} \\
& +\frac{g^{2}}{4} f^{a b c} f^{a d e} A_{b \mu} A^{d \mu} A_{c \nu} A^{e \nu} \\
& -\frac{g^{2}}{2} \bar{\psi}^{i} \gamma^{+}\left(\gamma^{\perp^{\prime}} A_{\perp^{\prime}}\right)^{i j} \frac{1}{i \partial_{-}}\left(\gamma^{\perp} A_{\perp}\right)^{j k} \psi^{k} \\
& -\frac{g^{2}}{2} j^{+}{ }_{a} \frac{1}{\left(\partial_{-}\right)^{2}} j^{+}{ }_{a}
\end{aligned}
$$

where

$$
j^{+}{ }_{a}=\bar{\psi}^{i} \gamma^{+}\left(t_{a}\right)^{i j} \psi^{j}+f_{a b c}\left(\partial_{-} A_{b \mu}\right) A^{c \mu}
$$

In light-cone time-ordered perturbation theory, a Green's functions is expanded as a power series in the interactions with light-front energy denominators $\sum_{\text {initial }} k_{i}^{-}-$ $\sum_{\text {intermediate }} k_{i}^{-}+i \epsilon$ replacing the usual energy denominators. [For a review see Ref. [16].] In general each Feynman diagram with $n$ vertices corresponds to the sum of $n$ ! time-ordered contributions. However, in light-cone-time-ordered perturbation theory, only those few graphs where all $k_{i}^{+} \geq 0$ survive. In addition the form of the light-front kinetic energies is rational: $k^{-}=\frac{k_{\perp}^{2}+m^{2}}{k^{+}}$, replacing the nonanalytic $k^{0}=\sqrt{\vec{k}^{2}+m^{2}}$ of equal-time theory. Thus light-cone-time-ordered perturbation theory provides a viable computational method where one can trace the physical evolution of a process. The integration measures are only three-dimensional $d^{2} k_{\perp} d x$; in effect, the $k^{-}$integral of the covariant perturbation theory is performed automatically.

Alternatively, one derive Feynman rules for QCD in light-cone gauge, thus allowing the use of standard covariant computational tools and renormalization methods including dimensional regularization. Prem Srivastava and I [18 have recently presented a systematic study of light-front-quantized gauge theory in light-cone gauge using a Dyson-Wick S-matrix expansion based on light-cone-time-ordered products. The gluon propagator has the form

$$
\left\langle 0\left|T\left(A^{a}{ }_{\mu}(x) A^{b}{ }_{\nu}(0)\right)\right| 0\right\rangle=\frac{i \delta^{a b}}{(2 \pi)^{4}} \int d^{4} k e^{-i k \cdot x} \frac{D_{\mu \nu}(k)}{k^{2}+i \epsilon}
$$


where we have defined

$$
D_{\mu \nu}(k)=D_{\nu \mu}(k)=-g_{\mu \nu}+\frac{n_{\mu} k_{\nu}+n_{\nu} k_{\mu}}{(n \cdot k)}-\frac{k^{2}}{(n \cdot k)^{2}} n_{\mu} n_{\nu} .
$$

Here $n_{\mu}$ is a null four-vector, gauge direction, whose components are chosen to be $n_{\mu}=\delta_{\mu}^{+}, n^{\mu}=\delta_{-}^{\mu}$. Note also

$$
\begin{aligned}
& D_{\mu \lambda}(k) D_{\nu}^{\lambda}(k)=D_{\mu \perp}(k) D_{\nu}^{\perp}(k)=-D_{\mu \nu}(k), \\
& k^{\mu} D_{\mu \nu}(k)=0, \quad n^{\mu} D_{\mu \nu}(k) \equiv D_{-\nu}(k)=0, \\
& D_{\lambda \mu}(q) D^{\mu \nu}(k) D_{\nu \rho}\left(q^{\prime}\right)=-D_{\lambda \mu}(q) D^{\mu \rho}\left(q^{\prime}\right) .
\end{aligned}
$$

The gauge field propagator $i D_{\mu \nu}(k) /\left(k^{2}+i \epsilon\right)$ is transverse not only to the gauge direction $n_{\mu}$ but also to $k_{\mu}$, i.e., it is doubly-transverse. This leads to appreciable simplifications in the computations in QCD. For example, the coupling of gluons to propagators carrying high momenta is automatic. The absence of collinear divergences in irreducible diagrams in the light-cone gauge greatly simplifies the leading-twist factorization of soft and hard gluonic corrections in high momentum transfer inclusive and exclusive reactions [5] since the numerators associated with the gluon coupling only have transverse components. The renormalization factors in the light-cone gauge are independent of the reference direction $n^{\mu}$. Since the gluon only has physical polarization, its renormalization factors satisfy $Z_{1}=Z_{3}$. Because of its explicit unitarity in each graph, the doubly-transverse gauge is well suited for calculations identifying the "pinch" effective charge [19, 20].

The running coupling constant and QCD $\beta$ function have also been computed at one loop in the doubly-transverse light-cone gauge [18. It is also possible to effectively quantize QCD using light-front methods in covariant Feynman gauge [21].

A remarkable advantage of light-front quantization is that the vacuum state $|0\rangle$ of the full QCD Hamiltonian evidently coincides with the free vacuum. The light-front vacuum is effectively trivial if the interaction Hamiltonian applied to the perturbative vacuum is zero. Note that all particles in the Hilbert space have positive energy $k^{0}=$ $\frac{1}{2}\left(k^{+}+k^{-}\right)$, and thus positive light-front $k^{ \pm}$. Since the plus momenta $\sum k_{i}^{+}$is conserved by the interactions, the perturbative vacuum can only couple to states with particles in which all $k_{i}^{+}=0$; i.e., so called zero-mode states. In the case of QED, a massive electron cannot have $k^{+}=0$ unless it also has infinite energy. In a remarkable calculation, Bassetto and collaborators 22 have shown that the computation of the spectrum of $Q C D(1+1)$ in equal time quantization requires constructing the full spectrum of non perturbative contributions (instantons). In contrast, in the light-front quantization of 
gauge theory, where the $k^{+}=0$ singularity of the instantaneous interaction is defined by a simple infrared regularization, one obtains the correct spectrum of $Q C D(1+1)$ without any need for vacuum-related contributions.

In the case of $\mathrm{QCD}(3+1)$, the momentum-independent four-gluon non-Abelian interaction in principle can couple the perturbative vacuum to a state with four collinear gluons in which all of the gluons have all components $k_{i}^{\mu}=0$, thus hinting at role for zero modes in theories with massless quanta. In fact, zero modes of auxiliary fields are necessary to distinguish the theta-vacua of massless $\mathrm{QED}(1+1)$ [23, 24, 25], or to represent a theory in the presence of static external boundary conditions or other constraints. Zero-modes provide the light-front representation of spontaneous symmetry breaking in scalar theories [26].

There are a number of other simplifications of the light-front formalism:

1. The light-front wavefunctions describe quanta which have positive energy, positive norm, and physical polarization. The formalism is thus physical, and unitary. No ghosts fields appear explicitly, even in non-Abelian theory. The wavefunctions are only functions of three rather than four physical momentum variables: the light-front momentum fractions $x_{i}$ and transverse momenta $k_{\perp}$. The quarks and gluons each have two physical polarization states.

2. The set of light-front wavefunctions provide a frame-independent, quantummechanical description of hadrons at the amplitude level capable of encoding multiquark and gluon momentum, helicity, and flavor correlations in the form of universal process-independent hadron wavefunctions. Matrix elements of spacelike currents such as the spacelike electromagnetic form factors have an exact representation in terms of simple overlaps of the light-front wavefunctions in momentum space with the same $x_{i}$ and unchanged parton number [27, 28, 29]. In the case of timelike decays, such as those determined by semileptonic $B$ decay, one needs to include contributions in which the parton number $\Delta n=2$. [30]. The leading-twist off-forward parton distributions measured in deeply virtual Compton scattering have a similar light-front wavefunction representation [31, 32].

3. The high $x \rightarrow 1$ and high $k_{\perp}$ limits of the hadron wavefunctions control processes and reactions in which the hadron wavefunctions are highly stressed. Such configurations involve far-off-shell intermediate states and can be systematically treated in perturbation theory [33, 5].

4. The leading-twist structure functions $q_{i}(x, Q)$ and $g(x, Q)$ measured in deep inelastic scattering can be computed from the absolute squares of the light-front wavefunctions, integrated over the transverse momentum up to the resolution scale $Q$. All 
helicity distributions are thus encoded in terms of the light-front wavefunctions. The DGLAP evolution of the structure functions can be derived from the high $k_{\perp}$ properties of the light-front wavefunctions. Thus given the light-front wavefunctions, one can compute [5] all of the leading twist helicity and transversity distributions measured in polarized deep inelastic lepton scattering. For example, the helicity-specific quark distributions at resolution $\Lambda$ correspond to

$$
\begin{aligned}
q_{\lambda_{q} / \Lambda_{p}}(x, \Lambda)= & \sum_{n, q_{a}} \int \prod_{j=1}^{n} \frac{d x_{j} d^{2} k_{\perp j}}{16 \pi^{3}} \sum_{\lambda_{i}}\left|\psi_{n / H}^{(\Lambda)}\left(x_{i}, \vec{k}_{\perp i}, \lambda_{i}\right)\right|^{2} \\
& \times 16 \pi^{3} \delta\left(1-\sum_{i}^{n} x_{i}\right) \delta^{(2)}\left(\sum_{i}^{n} \vec{k}_{\perp i}\right) \delta\left(x-x_{q}\right) \delta_{\lambda, \lambda_{q}} \Theta\left(\Lambda^{2}-\mathcal{M}_{n}^{2}\right),
\end{aligned}
$$

where the sum is over all quarks $q_{a}$ which match the quantum numbers, light-front momentum fraction $x$, and helicity of the struck quark. Similarly, the transversity distributions and off-diagonal helicity convolutions are defined as a density matrix of the light-front wavefunctions. This defines the LC factorization scheme [5] where the invariant mass squared $\mathcal{M}_{n}^{2}=\sum_{i=1}^{n}\left(k_{\perp i}^{2}+m_{i}^{2}\right) / x_{i}$ of the $n$ partons of the light-front wavefunctions is limited to $\mathcal{M}_{n}^{2}<\Lambda^{2}$

5. The distribution of spectator particles in the final state in the proton fragmentation region in deep inelastic scattering at an electron-proton collider are encoded in the light-front wavefunctions of the target proton. Conversely, the light-front wavefunctions can be used to describe the coalescence of comoving quarks into final state hadrons.

6. The light-front wavefunctions also specify the multi-quark and gluon correlations of the hadron. Despite the many sources of power-law corrections to the deep inelastic cross section, certain types of dynamical contributions will stand out at large $x_{b j}$ since they arise from compact, highly-correlated fluctuations of the proton wavefunction. In particular, there are particularly interesting dynamical $\mathcal{O}\left(1 / Q^{2}\right)$ corrections which are due to the interference of quark currents; i.e., contributions which involve leptons scattering amplitudes from two different quarks of the target nucleon [34].

7. The higher Fock states of the light hadrons describe the sea quark structure of the deep inelastic structure functions, including "intrinsic" strangeness and charm fluctuations specific to the hadron's structure rather than gluon substructure [35, 36]. Ladder relations connecting state of different particle number follow from the QCD equation of motion and lead to Regge behavior of the quark and gluon distributions at $x \rightarrow 0$ 37.

8. The gauge- and process-independent meson and baryon valence-quark distribution amplitudes $\phi_{M}(x, Q)$, and $\phi_{B}\left(x_{i}, Q\right)$ which control exclusive processes involving a 
hard scale $Q$, including heavy quark decays, are given by the valence light-front Fock state wavefunctions integrated over the transverse momentum up to the resolution scale $Q$. The evolution equations for distribution amplitudes follow from the perturbative high transverse momentum behavior of the light-front wavefunctions [16.

9. The line-integrals needed to defining distribution amplitudes and structure functions as gauge invariant matrix elements of operator products vanish in light-front gauge.

10. Proofs of factorization theorems in hard exclusive and inclusive reactions are greatly simplified since the propagating gluons in light-cone gauge couple only to transverse currents; collinear divergences are thus automatically suppressed.

11. At high energies each light-front Fock state interacts distinctly; e.g., Fock states with small particle number and small impact separation have small color dipole moments and can traverse a nucleus with minimal interactions. This is the basis for the predictions for "color transparency" in hard quasi-exclusive [38, 39] and diffractive reactions [2, 3, 国].

12. The Fock state wavefunctions of hadron can be resolved by a high energy diffractive interaction, producing forward jets with momenta which follow the lightfront momenta of the wavefunction [2, 3, 4].

13. The deuteron form factor at high $Q^{2}$ is sensitive to wavefunction configurations where all six quarks overlap within an impact separation $b_{\perp i}<\mathcal{O}(1 / Q)$. The leading power-law fall off predicted by QCD is $F_{d}\left(Q^{2}\right)=f\left(\alpha_{s}\left(Q^{2}\right)\right) /\left(Q^{2}\right)^{5}$, where, asymptotically, $f\left(\alpha_{s}\left(Q^{2}\right)\right) \propto \alpha_{s}\left(Q^{2}\right)^{5+2 \gamma} 40$, 41. In general, the six-quark wavefunction of a deuteron is a mixture of five different color-singlet states. The dominant color configuration at large distances corresponds to the usual proton-neutron bound state. However at small impact space separation, all five Fock color-singlet components eventually evolve to a state with equal weight, i.e., the deuteron wavefunction evolves to $80 \%$ "hidden color" 41. The relatively large normalization of the deuteron form factor observed at large $Q^{2}$ hints at sizable hidden-color contributions 42. Hidden color components can also play a predominant role in the reaction $\gamma d \rightarrow J / \psi p n$ at threshold if it is dominated by the multi-fusion process $\gamma g g \rightarrow J / \psi$ 43. Hard exclusive nuclear processes can also be analyzed in terms of "reduced amplitudes" which remove the effects of nucleon substructure.

Light-front wavefunctions are thus the frame-independent interpolating functions between hadron and quark and gluon degrees of freedom. Hadron amplitudes are computed from the convolution of the light-front wavefunctions with irreducible quarkgluon amplitudes. More generally, all multi-quark and gluon correlations in the bound 
state are represented by the light-front wavefunctions. The light-front Fock representation is thus a representation of the underlying quantum field theory. I will discuss progress in computing light-front wavefunctions directly from QCD in Sections 9 and 10.

\section{Other Theoretical Tools}

In addition to the light-front Fock expansion, a number of other useful theoretical tools are available to eliminate theoretical ambiguities in QCD predictions:

(1) Conformal symmetry provides a template for QCD predictions [44, leading to relations between observables which are present even in a theory which is not scale invariant. For example, the natural representation of distribution amplitudes is in terms of an expansion of orthonormal conformal functions multiplied by anomalous dimensions determined by QCD evolution equations 45, 46, 47. Thus an important guide in QCD analyses is to identify the underlying conformal relations of QCD which are manifest if we drop quark masses and effects due to the running of the QCD couplings. In fact, if QCD has an infrared fixed point (vanishing of the Gell Mann-Low function at low momenta), the theory will closely resemble a scale-free conformally symmetric theory in many applications.

(2) Commensurate scale relations 48, 49] are perturbative QCD predictions which relate observable to observable at fixed relative scale, such as the "generalized Crewther relation" [50], which connects the Bjorken and Gross-Llewellyn Smith deep inelastic scattering sum rules to measurements of the $e^{+} e^{-}$annihilation cross section. Such relations have no renormalization scale or scheme ambiguity. The coefficients in the perturbative series for commensurate scale relations are identical to those of conformal QCD; thus no infrared renormalons are present 44. One can identify the required conformal coefficients at any finite order by expanding the coefficients of the usual PQCD expansion around a formal infrared fixed point, as in the Banks-Zak method [20]. All non-conformal effects are absorbed by fixing the ratio of the respective momentum transfer and energy scales. In the case of fixed-point theories, commensurate scale relations relate both the ratio of couplings and the ratio of scales as the fixed point is approached 44.

(3) $\alpha_{V}$ and Skeleton Schemes. A physically natural scheme for defining the QCD coupling in exclusive and other processes is the $\alpha_{V}\left(Q^{2}\right)$ scheme defined from the potential of static heavy quarks. Heavy-quark lattice gauge theory can provide highly precise values for the coupling. All vacuum polarization corrections due to fermion pairs are 
then automatically and analytically incorporated into the Gell Mann-Low function, thus avoiding the problem of explicitly computing and resumming quark mass corrections related to the running of the coupling [51]. The use of a finite effective charge such as $\alpha_{V}$ as the expansion parameter also provides a basis for regulating the infrared nonperturbative domain of the QCD coupling. A similar coupling and scheme can be based on an assumed skeleton expansion of the theory [19, 20].

(4) The Abelian Correspondence Principle. One can consider QCD predictions as analytic functions of the number of colors $N_{C}$ and flavors $N_{F}$. In particular, one can show at all orders of perturbation theory that PQCD predictions reduce to those of an Abelian theory at $N_{C} \rightarrow 0$ with $\widehat{\alpha}=C_{F} \alpha_{s}$ and $\widehat{N}_{F}=2 N_{F} / C_{F}$ held fixed [52. There is thus a deep connection between QCD processes and their corresponding QED analogs.

\section{Applications of light-front Wavefunctions to Cur- rent Matrix Elements}

The light-front Fock representation of current matrix elements has a number of simplifying properties. The space-like local operators for the coupling of photons, gravitons and the deep inelastic structure functions can all be expressed as overlaps of light-front wavefunctions with the same number of Fock constituents. This is possible since one can choose the special frame $q^{+}=0$ [27, 28] for space-like momentum transfer and take matrix elements of "plus" components of currents such as $J^{+}$and $T^{++}$. No contributions to the current matrix elements from vacuum fluctuations occur. Similarly, given the local operators for the energy-momentum tensor $T^{\mu \nu}(x)$ and the angular momentum tensor $M^{\mu \nu \lambda}(x)$, one can directly compute momentum fractions, spin properties, and the form factors $A\left(q^{2}\right)$ and $B\left(q^{2}\right)$ appearing in the coupling of gravitons to composite systems [17].

In the case of a spin- $\frac{1}{2}$ composite system, the Dirac and Pauli form factors $F_{1}\left(q^{2}\right)$ and $F_{2}\left(q^{2}\right)$ are defined by

$$
\left\langle P^{\prime}\left|J^{\mu}(0)\right| P\right\rangle=\bar{u}\left(P^{\prime}\right)\left[F_{1}\left(q^{2}\right) \gamma^{\mu}+F_{2}\left(q^{2}\right) \frac{i}{2 M} \sigma^{\mu \alpha} q_{\alpha}\right] u(P)
$$

where $q^{\mu}=\left(P^{\prime}-P\right)^{\mu}$ and $u(P)$ is the bound state spinor. In the light-front formalism it is convenient to identify the Dirac and Pauli form factors from the helicity-conserving and helicity-flip vector current matrix elements of the $J^{+}$current [29]:

$$
\left\langle P+q, \uparrow\left|\frac{J^{+}(0)}{2 P^{+}}\right| P, \uparrow\right\rangle=F_{1}\left(q^{2}\right),
$$




$$
\left\langle P+q, \uparrow\left|\frac{J^{+}(0)}{2 P^{+}}\right| P, \downarrow\right\rangle=-\left(q^{1}-\mathrm{i} q^{2}\right) \frac{F_{2}\left(q^{2}\right)}{2 M} .
$$

The magnetic moment of a composite system is one of its most basic properties. The magnetic moment is defined at the $q^{2} \rightarrow 0$ limit,

$$
\mu=\frac{e}{2 M}\left[F_{1}(0)+F_{2}(0)\right],
$$

where $e$ is the charge and $M$ is the mass of the composite system. We use the standard light-front frame $\left(q^{ \pm}=q^{0} \pm q^{3}\right)$ :

$$
\begin{gathered}
q=\left(q^{+}, q^{-}, \vec{q}_{\perp}\right)=\left(0, \frac{-q^{2}}{P^{+}}, \vec{q}_{\perp}\right), \\
P=\left(P^{+}, P^{-}, \vec{P}_{\perp}\right)=\left(P^{+}, \frac{M^{2}}{P^{+}}, \overrightarrow{0}_{\perp}\right),
\end{gathered}
$$

where $q^{2}=-2 P \cdot q=-\vec{q}_{\perp}^{2}$ is 4-momentum square transferred by the photon.

The Pauli form factor and the anomalous magnetic moment $\kappa=\frac{e}{2 M} F_{2}(0)$ can then be calculated from the expression

$$
-\left(q^{1}-\mathrm{i} q^{2}\right) \frac{F_{2}\left(q^{2}\right)}{2 M}=\sum_{a} \int \frac{\mathrm{d}^{2} \vec{k}_{\perp} \mathrm{d} x}{16 \pi^{3}} \sum_{j} e_{j} \psi_{a}^{\uparrow *}\left(x_{i}, \vec{k}_{\perp i}^{\prime}, \lambda_{i}\right) \psi_{a}^{\downarrow}\left(x_{i}, \vec{k}_{\perp i}, \lambda_{i}\right)
$$

where the summation is over all contributing Fock states $a$ and struck constituent charges $e_{j}$. The arguments of the final-state light-front wavefunction are

$$
\vec{k}_{\perp i}^{\prime}=\vec{k}_{\perp i}+\left(1-x_{i}\right) \vec{q}_{\perp}
$$

for the struck constituent and

$$
\vec{k}_{\perp i}^{\prime}=\vec{k}_{\perp i}-x_{i} \vec{q}_{\perp}
$$

for each spectator. Notice that the magnetic moment must be calculated from the spinflip non-forward matrix element of the current. In the ultra-relativistic limit where the radius of the system is small compared to its Compton scale $1 / M$, the anomalous magnetic moment must vanish [29]. The light-front formalism is consistent with this theorem.

The form factors of the energy-momentum tensor for a spin- $\frac{1}{2}$ composite are defined by

$$
\begin{array}{r}
\left\langle P^{\prime}\left|T^{\mu \nu}(0)\right| P\right\rangle=\bar{u}\left(P^{\prime}\right)\left[A\left(q^{2}\right) \gamma^{(\mu} \bar{P}^{\nu)}+B\left(q^{2}\right) \frac{i}{2 M} \bar{P}^{(\mu} \sigma^{\nu) \alpha} q_{\alpha}\right. \\
\left.+C\left(q^{2}\right) \frac{1}{M}\left(q^{\mu} q^{\nu}-g^{\mu \nu} q^{2}\right)\right] u(P),
\end{array}
$$


where $q^{\mu}=\left(P^{\prime}-P\right)^{\mu}, \bar{P}^{\mu}=\frac{1}{2}\left(P^{\prime}+P\right)^{\mu}, a^{(\mu} b^{\nu)}=\frac{1}{2}\left(a^{\mu} b^{\nu}+a^{\nu} b^{\mu}\right)$, and $u(P)$ is the spinor of the system. One can also readily obtain the light-front representation of the $A\left(q^{2}\right)$ and $B\left(q^{2}\right)$ form factors of the energy-tensor Eq. (15) [17. In the interaction picture, only the non-interacting parts of the energy momentum tensor $T^{++}(0)$ need to be computed:

$$
\begin{gathered}
\left\langle P+q, \uparrow\left|\frac{T^{++}(0)}{2\left(P^{+}\right)^{2}}\right| P, \uparrow\right\rangle=A\left(q^{2}\right) \\
\left\langle P+q, \uparrow\left|\frac{T^{++}(0)}{2\left(P^{+}\right)^{2}}\right| P, \downarrow\right\rangle=-\left(q^{1}-\mathrm{i} q^{2}\right) \frac{B\left(q^{2}\right)}{2 M} .
\end{gathered}
$$

The $A\left(q^{2}\right)$ and $B\left(q^{2}\right)$ form factors Eqs. (16) and (17) are similar to the $F_{1}\left(q^{2}\right)$ and $F_{2}\left(q^{2}\right)$ form factors Eqs. (8) and (9) with an additional factor of the light-front momentum fraction $x=k^{+} / P^{+}$of the struck constituent in the integrand. The $B\left(q^{2}\right)$ form factor is obtained from the non-forward spin-flip amplitude. The value of $B(0)$ is obtained in the $q^{2} \rightarrow 0$ limit. The angular momentum projection of a state is given by

$$
\left\langle J^{i}\right\rangle=\frac{1}{2} \epsilon^{i j k} \int d^{3} x\left\langle T^{0 k} x^{j}-T^{0 j} x^{k}\right\rangle=A(0)\left\langle L^{i}\right\rangle+[A(0)+B(0)] \bar{u}(P) \frac{1}{2} \sigma^{i} u(P) .
$$

This result is derived using a wave-packet description of the state. The $\left\langle L^{i}\right\rangle$ term is the orbital angular momentum of the center of mass motion with respect to an arbitrary origin and can be dropped. The coefficient of the $\left\langle L^{i}\right\rangle$ term must be $1 ; A(0)=1$ also follows when we evaluate the four-momentum expectation value $\left\langle P^{\mu}\right\rangle$. Thus the total intrinsic angular momentum $J^{z}$ of a nucleon can be identified with the values of the form factors $A\left(q^{2}\right)$ and $B\left(q^{2}\right)$ at $q^{2}=0$ :

$$
\left\langle J^{z}\right\rangle=\left\langle\frac{1}{2} \sigma^{z}\right\rangle[A(0)+B(0)]
$$

The anomalous moment coupling $B(0)$ to a graviton is shown to vanish for any composite system. This remarkable result, first derived by Okun and Kobzarev [53, 54, 55, 56, 57, is shown to follow directly from the Lorentz boost properties of the light-front Fock representation [17].

Dae Sung Hwang, Bo-Qiang Ma, Ivan Schmidt, and I [17] have recently shown that the light-front wavefunctions generated by the radiative corrections to the electron in QED provides a simple system for understanding the spin and angular momentum decomposition of relativistic systems. This perturbative model also illustrates the interconnections between Fock states of different number. The model is patterned after the quantum structure which occurs in the one-loop Schwinger $\alpha / 2 \pi$ correction to the electron magnetic moment [29]. In effect, we can represent a spin- $\frac{1}{2}$ system as a composite of a spin- $\frac{1}{2}$ fermion and spin-one vector boson with arbitrary masses. A similar 
model has been used to illustrate the matrix elements and evolution of light-front helicity and orbital angular momentum operators [58]. This representation of a composite system is particularly useful because it is based on two constituents but yet is totally relativistic. We can then explicitly compute the form factors $F_{1}\left(q^{2}\right)$ and $F_{2}\left(q^{2}\right)$ of the electromagnetic current, and the various contributions to the form factors $A\left(q^{2}\right)$ and $B\left(q^{2}\right)$ of the energy-momentum tensor.
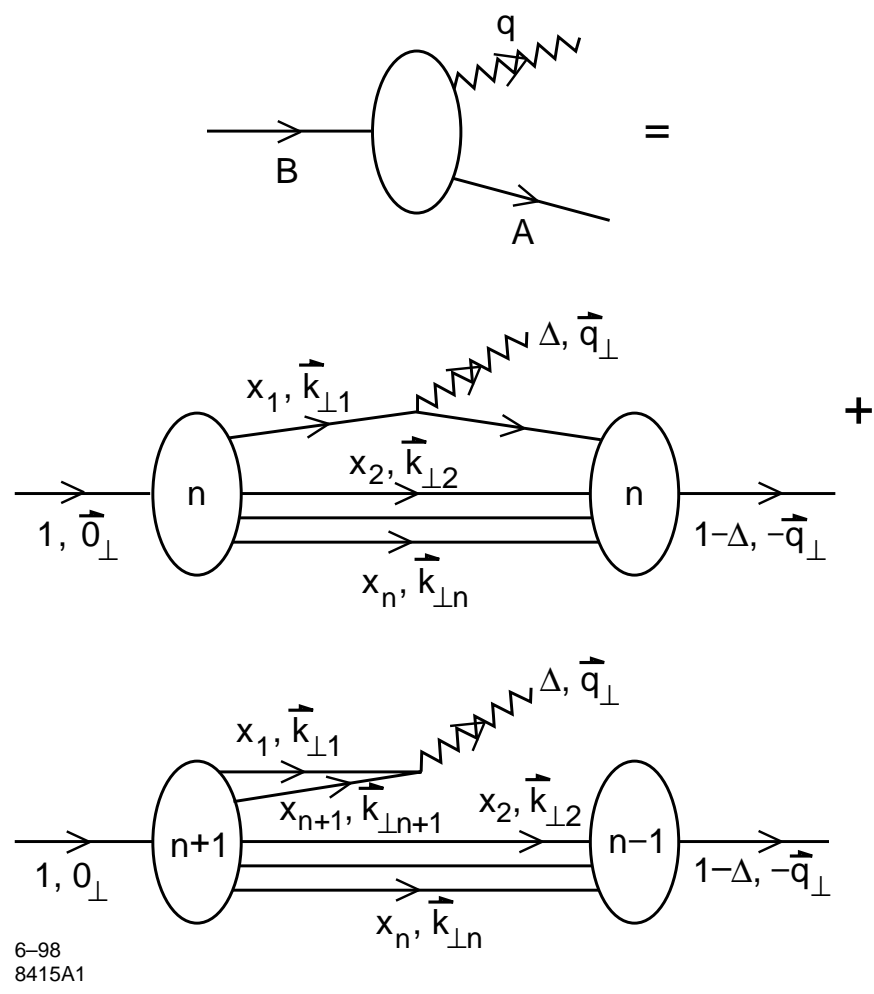

Figure 1: Exact representation of electroweak decays and time-like form factors in the light-front Fock representation.

Another remarkable advantage of the light-front formalism is that exclusive semileptonic $B$-decay amplitudes such as $B \rightarrow A \ell \bar{\nu}$ can also be evaluated exactly [30]. The time-like decay matrix elements require the computation of the diagonal matrix element $n \rightarrow n$ where parton number is conserved, and the off-diagonal $n+1 \rightarrow n-1$ convolution where the current operator annihilates a $q \overline{q^{\prime}}$ pair in the initial $B$ wavefunction. See Fig. 1. This term is a consequence of the fact that the time-like decay $q^{2}=\left(p_{\ell}+p_{\bar{\nu}}\right)^{2}>0$ requires a positive light-front momentum fraction $q^{+}>0$. Conversely for space-like currents, one can choose $q^{+}=0$, as in the Drell-Yan-West representation of the space-like 
electromagnetic form factors. However, as can be seen from the explicit analysis of the form factor in a perturbative model, the off-diagonal convolution can yield a nonzero $q^{+} / q^{+}$limiting form as $q^{+} \rightarrow 0$. This extra term appears specifically in the case of "bad" currents such as $J^{-}$in which the coupling to $q \bar{q}$ fluctuations in the light-front wavefunctions are favored. In effect, the $q^{+} \rightarrow 0$ limit generates $\delta(x)$ contributions as residues of the $n+1 \rightarrow n-1$ contributions. The necessity for such "zero mode" $\delta(x)$ terms has been noted by Chang, Root and Yan [59], Burkardt [60], and Ji and Choi 61 .

The off-diagonal $n+1 \rightarrow n-1$ contributions give a new perspective for the physics of $B$-decays. A semileptonic decay involves not only matrix elements where a quark changes flavor, but also a contribution where the leptonic pair is created from the annihilation of a $q \overline{q^{\prime}}$ pair within the Fock states of the initial $B$ wavefunction. The semileptonic decay thus can occur from the annihilation of a nonvalence quark-antiquark pair in the initial hadron. This feature will carry over to exclusive hadronic $B$-decays, such as $B^{0} \rightarrow \pi^{-} D^{+}$. In this case the pion can be produced from the coalescence of a $d \bar{u}$ pair emerging from the initial higher particle number Fock wavefunction of the $B$. The $D$ meson is then formed from the remaining quarks after the internal exchange of a $W$ boson.

In principle, a precise evaluation of the hadronic matrix elements needed for $B$ decays and other exclusive electroweak decay amplitudes requires knowledge of all of the light-front Fock wavefunctions of the initial and final state hadrons. In the case of model gauge theories such as $\mathrm{QCD}(1+1)$ 62 or collinear QCD 63 in one-space and one-time dimensions, the complete evaluation of the light-front wavefunction is possible for each baryon or meson bound-state using the DLCQ method. It would be interesting to use such solutions as a model for physical $B$-decays.

\section{Light-front Representation of Deeply Virtual Comp- ton Scattering}

The virtual Compton scattering process $\frac{d \sigma}{d t}\left(\gamma^{*} p \rightarrow \gamma p\right)$ for large initial photon virtuality $q^{2}=-Q^{2}$ has extraordinary sensitivity to fundamental features of the proton's structure. Even though the final state photon is on-shell, the deeply virtual process probes the elementary quark structure of the proton near the light cone as an effective local current. In contrast to deep inelastic scattering, which measures only the absorptive part of the forward virtual Compton amplitude $\operatorname{Im} \mathcal{T}_{\gamma^{*} p \rightarrow \gamma^{*} p}$, deeply vir- 
tual Compton scattering allows the measurement of the phase and spin structure of proton matrix elements for general momentum transfer squared $t$. In addition, the interference of the virtual Compton amplitude and Bethe-Heitler wide angle scattering Bremsstrahlung amplitude where the photon is emitted from the lepton line leads to an electron-positron asymmetry in the $e^{ \pm} p \rightarrow e^{ \pm} \gamma p$ cross section which is proportional to the real part of the Compton amplitude [64, 65, 66]. The deeply virtual Compton amplitude $\gamma^{*} p \rightarrow \gamma p$ is related by crossing to another important process $\gamma^{*} \gamma \rightarrow$ hadron pairs at fixed invariant mass which can be measured in electron-photon collisions [67.

To leading order in $1 / Q$, the deeply virtual Compton scattering amplitude $\gamma^{*}(q) p(P) \rightarrow$ $\gamma\left(q^{\prime}\right) p\left(P^{\prime}\right)$ factorizes as the convolution in $x$ of the amplitude $t^{\mu \nu}$ for hard Compton scattering on a quark line with the generalized Compton form factors $H(x, t, \zeta), E(x, t, \zeta)$, $\tilde{H}(x, t, \zeta)$, and $\tilde{E}(x, t, \zeta)$ of the target proton [54, 55, 68, 69, 70, 71, 72, 73, 74, 75, 76, 77]. Here $x$ is the light-front momentum fraction of the struck quark, and $\zeta=Q^{2} / 2 P \cdot q$ plays the role of the Bjorken variable. The square of the four-momentum transfer from the proton is given by $t=\Delta^{2}=2 P \cdot \Delta=-\frac{\left(\zeta^{2} M^{2}+\vec{\Delta}_{\perp}^{2}\right)}{(1-\zeta)}$, where $\Delta$ is the difference of initial and final momenta of the proton $\left(P=P^{\prime}+\Delta\right)$. We will be interested in deeply virtual Compton scattering where $q^{2}$ is large compared to the masses and $t$. Then, to leading order in $1 / Q^{2}, \frac{-q^{2}}{2 P_{I} \cdot q}=\zeta$. Thus $\zeta$ plays the role of the Bjorken variable in deeply virtual Compton scattering. For a fixed value of $-t$, the allowed range of $\zeta$ is given by

$$
0 \leq \zeta \leq \frac{(-t)}{2 M^{2}}\left(\sqrt{1+\frac{4 M^{2}}{(-t)}}-1\right)
$$

The form factor $H(x, t, \zeta)$ describes the proton response when the helicity of the proton is unchanged, and $E(x, t, \zeta)$ is for the case when the proton helicity is flipped. Two additional functions $\tilde{H}(x, t, \zeta)$, and $\tilde{E}(x, t, \zeta)$ appear, corresponding to the dependence of the Compton amplitude on quark helicity.

Recently, Markus Diehl, Dae Sung Hwang and I [31] have shown how the deeply virtual Compton amplitude can be evaluated explicitly in the Fock state representation using the matrix elements of the currents and the boost properties of the light-front wavefunctions. For the $n \rightarrow n$ diagonal term $(\Delta n=0)$, the arguments of the final-state hadron wavefunction are $\frac{x_{1}-\zeta}{1-\zeta}, \vec{k}_{\perp 1}-\frac{1-x_{1}}{1-\zeta} \vec{\Delta}_{\perp}$ for the struck quark and $\frac{x_{i}}{1-\zeta}, \vec{k}_{\perp i}+\frac{x_{i}}{1-\zeta} \vec{\Delta}_{\perp}$ for the $n-1$ spectators. We thus obtain formulae for the diagonal (parton-numberconserving) contribution to the generalized form factors for deeply virtual Compton amplitude in the domain [75, 74, 78] $\zeta \leq x_{1} \leq 1$ : 

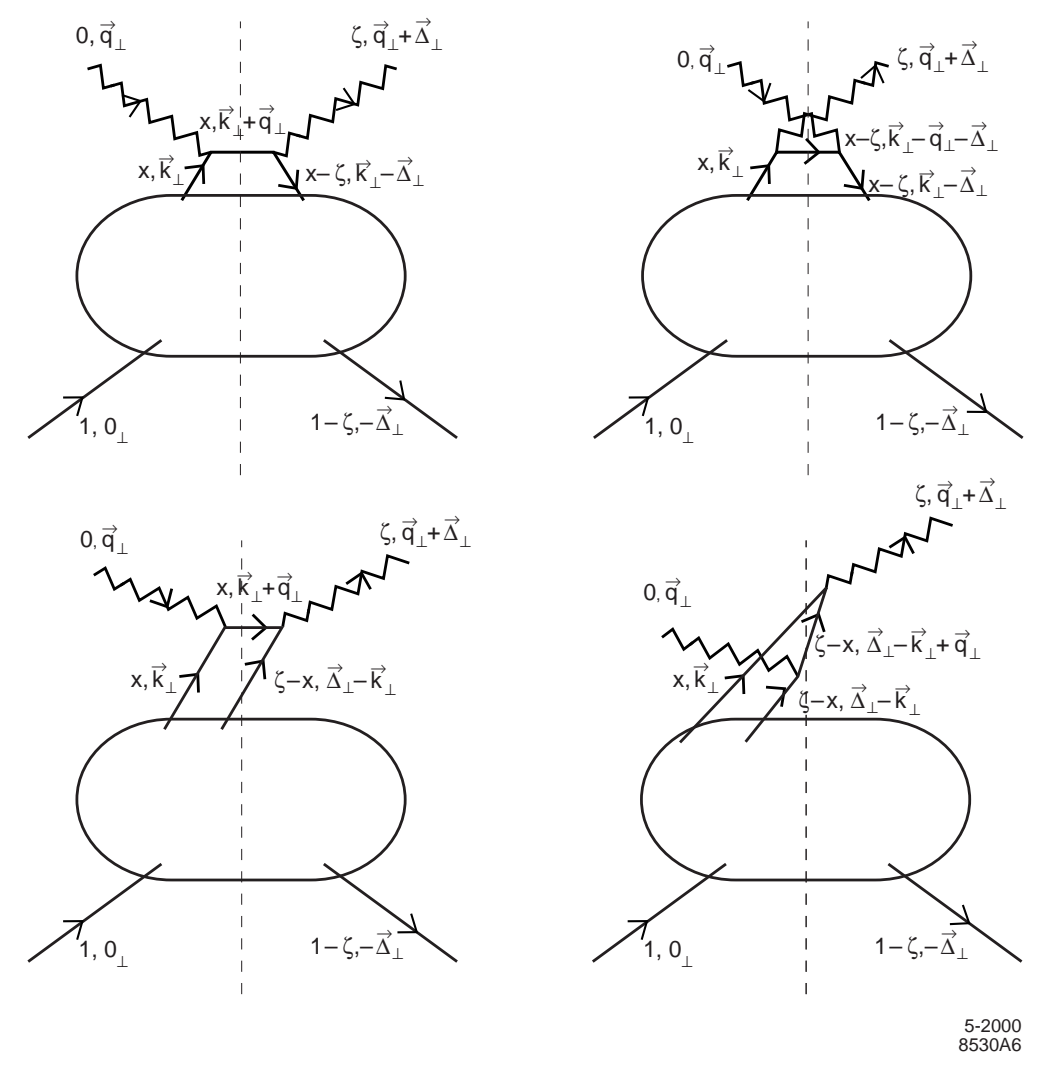

Figure 2: Light-cone time-ordered contributions to deeply virtual Compton scattering. Only the contributions of leading twist in $1 / q^{2}$ are illustrated. These contributions illustrate the factorization property of the leading twist amplitude.

$$
\begin{aligned}
& \sqrt{1-\zeta} f_{1(n \rightarrow n)}\left(x_{1}, t, \zeta\right)-\frac{\zeta^{2}}{4 \sqrt{1-\zeta}} f_{2(n \rightarrow n)}\left(x_{1}, t, \zeta\right) \\
&= \sum_{n, \lambda} \prod_{i=1}^{n} \int_{0}^{1} d x_{i(i \neq 1)} \int \frac{d^{2} \vec{k}_{\perp i}}{2(2 \pi)^{3}} \delta\left(1-\sum_{j=1}^{n} x_{j}\right) \delta^{(2)}\left(\sum_{j=1}^{n} \vec{k}_{\perp j}\right) \\
& \times \psi_{(n)}^{\uparrow *}\left(x_{i}^{\prime}, \vec{k}_{\perp i}^{\prime}, \lambda_{i}\right) \psi_{(n)}^{\uparrow}\left(x_{i}, \vec{k}_{\perp i}, \lambda_{i}\right)(\sqrt{1-\zeta})^{1-n} \\
& \sqrt{1-\zeta}\left(1+\frac{\zeta}{2(1-\zeta)}\right) \frac{\left(\Delta^{1}-\mathrm{i} \Delta^{2}\right)}{2 M} f_{2(n \rightarrow n)}\left(x_{1}, t, \zeta\right) \\
&= \sum_{n, \lambda} \prod_{i=1}^{n} \int_{0}^{1} d x_{i(i \neq 1)} \int \frac{d^{2} \vec{k}_{\perp i}}{2(2 \pi)^{3}} \delta\left(1-\sum_{j=1}^{n} x_{j}\right) \delta^{(2)}\left(\sum_{j=1}^{n} \vec{k}_{\perp j}\right) \\
& \times \psi_{(n)}^{\uparrow *}\left(x_{i}^{\prime}, \vec{k}_{\perp i}^{\prime}, \lambda_{i}\right) \psi_{(n)}^{\downarrow}\left(x_{i}, \vec{k}_{\perp i}, \lambda_{i}\right)(\sqrt{1-\zeta})^{1-n},
\end{aligned}
$$


where

$$
\begin{cases}x_{1}^{\prime}=\frac{x_{1}-\zeta}{1-\zeta}, & \vec{k}_{\perp 1}^{\prime}=\vec{k}_{\perp 1}-\frac{1-x_{1}}{1-\zeta} \vec{\Delta}_{\perp} \text { for the struck quark, } \\ x_{i}^{\prime}=\frac{x_{i}}{1-\zeta}, & \vec{k}_{\perp i}^{\prime}=\vec{k}_{\perp i}+\frac{x_{i}}{1-\zeta} \vec{\Delta}_{\perp} \quad \text { for the }(n-1) \text { spectators. }\end{cases}
$$

A sum over all possible helicities $\lambda_{i}$ is understood. If quark masses are neglected, the currents conserve helicity. We also can check that $\sum_{i=1}^{n} x_{i}^{\prime}=1, \sum_{i=1}^{n} \vec{k}_{\perp i}^{\prime}=\overrightarrow{0}_{\perp}$.

For the $n+1 \rightarrow n-1$ off-diagonal term $(\Delta n=-2)$, consider the case where partons 1 and $n+1$ of the initial wavefunction annihilate into the current leaving $n-1$ spectators. Then $x_{n+1}=\zeta-x_{1}, \vec{k}_{\perp n+1}=\vec{\Delta}_{\perp}-\vec{k}_{\perp 1}$. The remaining $n-1$ partons have total momentum $\left((1-\zeta) P^{+},-\vec{\Delta}_{\perp}\right)$. The final wavefunction then has arguments $x_{i}^{\prime}=\frac{x_{i}}{1-\zeta}$ and $\vec{k}_{\perp i}^{\prime}=\vec{k}_{\perp i}+\frac{x_{i}}{1-\zeta} \vec{\Delta}_{\perp}$. We thus obtain the formulae for the off-diagonal matrix element of the Compton amplitude in the domain $0 \leq x_{1} \leq \zeta$ :

$$
\begin{gathered}
\sqrt{1-\zeta} f_{1(n+1 \rightarrow n-1)}\left(x_{1}, t, \zeta\right)-\frac{\zeta^{2}}{4 \sqrt{1-\zeta}} f_{2(n+1 \rightarrow n-1)}\left(x_{1}, t, \zeta\right) \\
=\sum_{n, \lambda} \int_{0}^{1} d x_{n+1} \int \frac{d^{2} \vec{k}_{\perp 1}}{2(2 \pi)^{3}} \int \frac{d^{2} \vec{k}_{\perp n+1}}{2(2 \pi)^{3}} \prod_{i=2}^{n} \int_{0}^{1} d x_{i} \int \frac{d^{2} \vec{k}_{\perp i}}{2(2 \pi)^{3}} \\
\times \delta\left(1-\sum_{j=1}^{n+1} x_{j}\right) \delta^{(2)}\left(\sum_{j=1}^{n+1} \vec{k}_{\perp j}\right)[\sqrt{1-\zeta}]^{1-n} \\
\times \psi_{(n-1)}^{\uparrow *}\left(x_{i}^{\prime}, \vec{k}_{\perp i}^{\prime}, \lambda_{i}\right) \psi_{(n+1)}^{\uparrow}\left(\left\{x_{1}, x_{i}, x_{n+1}=\zeta-x_{1}\right\},\right. \\
\left.\left\{\vec{k}_{\perp 1}, \vec{k}_{\perp i}, \vec{k}_{\perp n+1}=\vec{\Delta}_{\perp}-\vec{k}_{\perp 1}\right\},\left\{\lambda_{1}, \lambda_{i}, \lambda_{n+1}=-\lambda_{1}\right\}\right), \\
\sqrt{1-\zeta}\left(1+\frac{\zeta}{2(1-\zeta)}\right) \frac{\left(\Delta^{1}-\mathrm{i} \Delta^{2}\right)}{2 M} f_{2(n+1 \rightarrow n-1)}\left(x_{1}, t, \zeta\right) \\
=\sum_{n, \lambda} \int_{0}^{1} d x_{n+1} \int \frac{d^{2} \vec{k}_{\perp 1}}{2(2 \pi)^{3}} \int \frac{d^{2} \vec{k}_{\perp n+1}}{2(2 \pi)^{3}} \prod_{i=2}^{n} \int_{0}^{1} d x_{i} \int \frac{d^{2} \vec{k}_{\perp i}}{2(2 \pi)^{3}} \\
\times \delta\left(1-\sum_{j=1}^{n+1} x_{j}\right) \delta^{(2)}\left(\sum_{j=1}^{n+1} \vec{k}_{\perp j}\right)[\sqrt{1-\zeta}]^{1-n} \\
\quad \times \psi_{(n-1)}^{\uparrow *}\left(x_{i}^{\prime}, \vec{k}_{\perp i}^{\prime}, \lambda_{i}\right) \psi_{(n+1)}^{\downarrow}\left(\left\{x_{1}, x_{i}, x_{n+1}=\zeta-x_{1}\right\}\right. \\
\left.\left\{\vec{k}_{\perp 1}, \vec{k}_{\perp i}, \vec{k}_{\perp n+1}=\vec{\Delta}_{\perp}-\vec{k}_{\perp 1}\right\},\left\{\lambda_{1}, \lambda_{i}, \lambda_{n+1}=-\lambda_{1}\right\}\right),
\end{gathered}
$$

where $i=2,3, \cdots, n$ label the $n-1$ spectator partons which appear in the final-state 
hadron wavefunction with

$$
x_{i}^{\prime}=\frac{x_{i}}{1-\zeta}, \quad \vec{k}_{\perp i}^{\prime}=\vec{k}_{\perp i}+\frac{x_{i}}{1-\zeta} \vec{\Delta}_{\perp}
$$

We can again check that the arguments of the final-state wavefunction satisfy $\sum_{i=2}^{n} x_{i}^{\prime}=$ $1, \sum_{i=2}^{n} \vec{k}_{\perp i}^{\prime}=\overrightarrow{0}_{\perp}$.

The above representation is the general form for the generalized form factors of the deeply virtual Compton amplitude for any composite system. Thus given the light-front Fock state wavefunctions of the eigensolutions of the light-front Hamiltonian, we can compute the amplitude for virtual Compton scattering including all spin correlations. The formulae are accurate to leading order in $1 / Q^{2}$. Radiative corrections to the quark Compton amplitude of order $\alpha_{s}\left(Q^{2}\right)$ from diagrams in which a hard gluon interacts between the two photons have also been neglected.

\section{Applications of QCD Factorization to Hard QCD Processes}

Factorization theorems for hard exclusive, semi-exclusive, and diffractive processes allow the separation of soft non-perturbative dynamics of the bound state hadrons from the hard dynamics of a perturbatively-calculable quark-gluon scattering amplitude. The factorization of inclusive reactions is reviewed in ref. For reviews and bibliography of exclusive process calculations in QCD (see Ref. [16, 79]).

The light-front formalism provides a physical factorization scheme which conveniently separates and factorizes soft non-perturbative physics from hard perturbative dynamics in both exclusive and inclusive reactions [5, 80].

In hard inclusive reactions all intermediate states are divided according to $\mathcal{M}_{n}^{2}<\Lambda^{2}$ and $\mathcal{M}_{n}^{2}>\Lambda^{2}$ domains. The lower mass regime is associated with the quark and gluon distributions defined from the absolute squares of the $\mathrm{LC}$ wavefunctions in the light cone factorization scheme. In the high invariant mass regime, intrinsic transverse momenta can be ignored, so that the structure of the process at leading power has the form of hard scattering on collinear quark and gluon constituents, as in the parton model. The attachment of gluons from the LC wavefunction to a propagator in a hard subprocess is power-law suppressed in LC gauge, so that the minimal quark-gluon particle-number subprocesses dominate. It is then straightforward to derive the DGLAP equations from the evolution of the distributions with $\log \Lambda^{2}$. The anomaly contribution to singlet helicity structure function $g_{1}(x, Q)$ can be explicitly identified in the LC factorization 
scheme as due to the $\gamma^{*} g \rightarrow q \bar{q}$ fusion process. The anomaly contribution would be zero if the gluon is on shell. However, if the off-shellness of the state is larger than the quark pair mass, one obtains the usual anomaly contribution [81].

In exclusive amplitudes, the LC wavefunctions are the interpolating amplitudes connecting the quark and gluons to the hadronic states. In an exclusive amplitude involving a hard scale $Q^{2}$ all intermediate states can be divided according to $\mathcal{M}_{n}^{2}<$ $\Lambda^{2}<Q^{2}$ and $\mathcal{M}_{n}^{2}<\Lambda^{2}$ invariant mass domains. The high invariant mass contributions to the amplitude has the structure of a hard scattering process $T_{H}$ in which the hadrons are replaced by their respective (collinear) quarks and gluons. In light-cone gauge only the minimal Fock states contribute to the leading power-law fall-off of the exclusive amplitude. The wavefunctions in the lower invariant mass domain can be integrated up to an arbitrary intermediate invariant mass cutoff $\Lambda$. The invariant mass domain beyond this cutoff is included in the hard scattering amplitude $T_{H}$. The $T_{H}$ satisfy dimensional counting rules [82]. Final-state and initial state corrections from gluon attachments to lines connected to the color-singlet distribution amplitudes cancel at leading twist. Explicit examples of perturbative QCD factorization will be discussed in more detail in the next section.

The key non-perturbative input for exclusive processes is thus the gauge and frame independent hadron distribution amplitude [80, 5] defined as the integral of the valence (lowest particle number) Fock wavefunction; e.g. for the pion

$$
\phi_{\pi}\left(x_{i}, \Lambda\right) \equiv \int d^{2} k_{\perp} \psi_{q \bar{q} / \pi}^{(\Lambda)}\left(x_{i}, \vec{k}_{\perp i}, \lambda\right)
$$

where the global cutoff $\Lambda$ is identified with the resolution $Q$. The distribution amplitude controls leading-twist exclusive amplitudes at high momentum transfer, and it can be related to the gauge-invariant Bethe-Salpeter wavefunction at equal light-cone time. The logarithmic evolution of hadron distribution amplitudes $\phi_{H}\left(x_{i}, Q\right)$ can be derived from the perturbatively-computable tail of the valence light-front wavefunction in the high transverse momentum regime [80, 0]. The conformal basis for the evolution of the three-quark distribution amplitudes for the baryons 83 has recently been obtained by V. Braun et al. 47.

The existence of an exact formalism provides a basis for systematic approximations and a control over neglected terms. For example, one can analyze exclusive semileptonic $B$-decays which involve hard internal momentum transfer using a perturbative QCD formalism [84, 85, 6, 7, 86, 87] patterned after the perturbative analysis of form factors at large momentum transfer. The hard-scattering analysis again proceeds by 
writing each hadronic wavefunction as a sum of soft and hard contributions

$$
\psi_{n}=\psi_{n}^{\text {soft }}\left(\mathcal{M}_{n}^{2}<\Lambda^{2}\right)+\psi_{n}^{\text {hard }}\left(\mathcal{M}_{n}^{2}>\Lambda^{2}\right)
$$

where $\mathcal{M}_{n}^{2}$ is the invariant mass of the partons in the $n$-particle Fock state and $\Lambda$ is the separation scale. The high internal momentum contributions to the wavefunction

$\psi_{n}^{\text {hard }}$ can be calculated systematically from QCD perturbation theory by iterating the gluon exchange kernel. The contributions from high momentum transfer exchange to the $B$-decay amplitude can then be written as a convolution of a hard-scattering quarkgluon scattering amplitude $T_{H}$ with the distribution amplitudes $\phi\left(x_{i}, \Lambda\right)$, the valence wavefunctions obtained by integrating the constituent momenta up to the separation scale $\mathcal{M}_{n}<\Lambda<Q$. Furthermore in processes such as $B \rightarrow \pi D$ where the pion is effectively produced as a rapidly-moving small Fock state with a small color-dipole interactions, final state interactions are suppressed by color transparency. This is the basis for the perturbative hard-scattering analyses [84, 6, 77, 86, 87]. In a systematic analysis, one can identify the hard PQCD contribution as well as the soft contribution from the convolution of the light-front wavefunctions. Furthermore, the hard-scattering contribution can be systematically improved.

Given the solution for the hadronic wavefunctions $\psi_{n}^{(\Lambda)}$ with $\mathcal{M}_{n}^{2}<\Lambda^{2}$, one can construct the wavefunction in the hard regime with $\mathcal{M}_{n}^{2}>\Lambda^{2}$ using projection operator techniques. The construction can be done perturbatively in QCD since only high invariant mass, far off-shell matrix elements are involved. One can use this method to derive the physical properties of the $\mathrm{LC}$ wavefunctions and their matrix elements at high invariant mass. Since $\mathcal{M}_{n}^{2}=\sum_{i=1}^{n}\left(\frac{k_{\perp}^{2}+m^{2}}{x}\right)_{i}$, this method also allows the derivation of the asymptotic behavior of light-front wavefunctions at large $k_{\perp}$, which in turn leads to predictions for the fall-off of form factors and other exclusive matrix elements at large momentum transfer, such as the quark counting rules for predicting the nominal powerlaw fall-off of two-body scattering amplitudes at fixed $\theta_{c m}$ [82] and helicity selection rules 88]. The phenomenological successes of these rules can be understood within QCD if the coupling $\alpha_{V}(Q)$ freezes in a range of relatively small momentum transfer 89.

\section{Two-Photon Processes}

The simplest and perhaps the most elegant illustration of an exclusive reaction in QCD is the evaluation of the photon-to-pion transition form factor $F_{\gamma \rightarrow \pi}\left(Q^{2}\right)$ [5, 90] which is measurable in single-tagged two-photon $e e \rightarrow e e \pi^{0}$ reactions. The form factor 
is defined via the invariant amplitude $\Gamma^{\mu}=-i e^{2} F_{\pi \gamma}\left(Q^{2}\right) \epsilon^{\mu \nu \rho \sigma} p_{\nu}^{\pi} \epsilon_{\rho} q_{\sigma}$. As in inclusive reactions, one must specify a factorization scheme which divides the integration regions of the loop integrals into hard and soft momenta, compared to the resolution scale $\tilde{Q}$. At leading twist, the transition form factor then factorizes as a convolution of the $\gamma^{*} \gamma \rightarrow q \bar{q}$ amplitude (where the quarks are collinear with the final state pion) with the valence light-front wavefunction of the pion:

$$
F_{\gamma M}\left(Q^{2}\right)=\frac{4}{\sqrt{3}} \int_{0}^{1} d x \phi_{M}(x, \tilde{Q}) T_{\gamma \rightarrow M}^{H}\left(x, Q^{2}\right) .
$$

The hard scattering amplitude for $\gamma \gamma^{*} \rightarrow q \bar{q}$ is $T_{\gamma M}^{H}\left(x, Q^{2}\right)=\left[(1-x) Q^{2}\right]^{-1} \times$ $\left(1+\mathcal{O}\left(\alpha_{s}\right)\right)$. The leading QCD corrections have been computed by Braaten [91]. The evaluation of the next-to-leading corrections in the physical $\alpha_{V}$ scheme is given in Ref. [89]. For the asymptotic distribution amplitude $\phi_{\pi}^{\text {asympt }}(x)=\sqrt{3} f_{\pi} x(1-x)$ one predicts $Q^{2} F_{\gamma \pi}\left(Q^{2}\right)=2 f_{\pi}\left(1-\frac{5}{3} \frac{\alpha_{V}\left(Q^{*}\right)}{\pi}\right)$ where $Q^{*}=e^{-3 / 2} Q$ is the BLM scale for the pion form factor. The PQCD predictions have been tested in measurements of $e \gamma \rightarrow e \pi^{0}$ by the CLEO collaboration 92. See Fig. 田 (b). The observed flat scaling of the $Q^{2} F_{\gamma \pi}\left(Q^{2}\right)$ data from $Q^{2}=2$ to $Q^{2}=8 \mathrm{GeV}^{2}$ provides an important confirmation of the applicability of leading twist QCD to this process. The magnitude of $Q^{2} F_{\gamma \pi}\left(Q^{2}\right)$ is remarkably consistent with the predicted form, assuming the asymptotic distribution amplitude and including the LO QCD radiative correction with $\alpha_{V}\left(e^{-3 / 2} Q\right) / \pi \simeq 0.12$. One could allow for some broadening of the distribution amplitude with a corresponding increase in the value of $\alpha_{V}$ at small scales. Radyushkin 93, Ong 94, and Kroll 95, have also noted that the scaling and normalization of the photon-to-pion transition form factor tends to favor the asymptotic form for the pion distribution amplitude and rules out broader distributions such as the two-humped form suggested by QCD sum rules [96].

The two-photon annihilation process $\gamma^{*} \gamma \rightarrow$ hadrons, which is measurable in singletagged $e^{+} e^{-} \rightarrow e^{+} e^{-}$hadrons events, provides a semi-local probe of $C=+$ hadron systems $\pi^{0}, \eta^{0}, \eta^{\prime}, \eta_{c}, \pi^{+} \pi^{-}$, etc. The $\gamma^{*} \gamma \rightarrow \pi^{+} \pi^{-}$hadron pair process is related to virtual Compton scattering on a pion target by crossing. The leading twist amplitude is sensitive to the $1 / x-1 /(1-x)$ moment of the two-pion distribution amplitude coupled to two valence quarks [78, 67].

Two-photon reactions, $\gamma \gamma \rightarrow H \bar{H}$ at large $\mathrm{s}=\left(k_{1}+k_{2}\right)^{2}$ and fixed $\theta_{\mathrm{cm}}$, provide a particularly important laboratory for testing QCD since these cross-channel "Compton" processes are the simplest calculable large-angle exclusive hadronic scattering reactions. The helicity structure, and often even the absolute normalization can be rigorously computed for each two-photon channel [90. In the case of meson pairs, dimensional counting predicts that for large $s, s^{4} d \sigma / d t\left(\gamma \gamma \rightarrow M \bar{M}\right.$ scales at fixed $t / s$ or $\theta_{\text {c.m. }}$ up to 
factors of $\ln s / \Lambda^{2}$. The angular dependence of the $\gamma \gamma \rightarrow H \bar{H}$ amplitudes can be used to determine the shape of the process-independent distribution amplitudes, $\phi_{H}(x, Q)$. An important feature of the $\gamma \gamma \rightarrow M \bar{M}$ amplitude for meson pairs is that the contributions of Landshoff pitch singularities are power-law suppressed at the Born level - even before taking into account Sudakov form factor suppression. There are also no anomalous contributions from the $x \rightarrow 1$ endpoint integration region. Thus, as in the calculation of the meson form factors, each fixed-angle helicity amplitude can be written to leading order in $1 / Q$ in the factorized form $\left[Q^{2}=p_{T}^{2}=t u / s ; \tilde{Q}_{x}=\min (x Q,(l-x) Q)\right]$ :

$$
\mathcal{M}_{\gamma \gamma \rightarrow M \bar{M}}=\int_{0}^{1} d x \int_{0}^{1} d y \phi_{\bar{M}}\left(y, \tilde{Q}_{y}\right) T_{H}\left(x, y, s, \theta_{\text {c.m. }} \phi_{M}\left(x, \tilde{Q}_{x}\right),\right.
$$

where $T_{H}$ is the hard-scattering amplitude $\gamma \gamma \rightarrow(q \bar{q})(q \bar{q})$ for the production of the valence quarks collinear with each meson, and $\phi_{M}(x, \tilde{Q})$ is the amplitude for finding the valence $q$ and $\bar{q}$ with light-front fractions of the meson's momentum, integrated over transverse momenta $k_{\perp}<\tilde{Q}$. The contribution of non-valence Fock states are power-law suppressed. Furthermore, the helicity-selection rules [88] of perturbative QCD predict that vector mesons are produced with opposite helicities to leading order in $1 / Q$ and all orders in $\alpha_{s}$. The dependence in $x$ and $y$ of several terms in $T_{\lambda, \lambda^{\prime}}$ is quite similar to that appearing in the meson's electromagnetic form factor. Thus much of the dependence on $\phi_{M}(x, Q)$ can be eliminated by expressing it in terms of the meson form factor. In fact, the ratio of the $\gamma \gamma \rightarrow \pi^{+} \pi^{-}$and $e^{+} e^{-} \rightarrow \mu^{+} \mu^{-}$amplitudes at large $s$ and fixed $\theta_{C M}$ is nearly insensitive to the running coupling and the shape of the pion distribution amplitude:

$$
\frac{\frac{d \sigma}{d t}\left(\gamma \gamma \rightarrow \pi^{+} \pi^{-}\right)}{\frac{d \sigma}{d t}\left(\gamma \gamma \rightarrow \mu^{+} \mu^{-}\right)} \sim \frac{4\left|F_{\pi}(s)\right|^{2}}{1-\cos ^{2} \theta_{\text {c.m. }}}
$$

The comparison of the PQCD prediction for the sum of $\pi^{+} \pi^{-}$plus $K^{+} K^{-}$channels with recent CLEO data [103] is shown in Fig. 3. The CLEO data for charged pion and kaon pairs show a clear transition to the scaling and angular distribution predicted by PQCD 90] for $W=\sqrt{(} s_{\gamma \gamma}>2 \mathrm{GeV}$. See Fig. 3. It is clearly important to measure the magnitude and angular dependence of the two-photon production of neutral pions and $\rho^{+} \rho^{-}$cross sections in view of the strong sensitivity of these channels to the shape of meson distribution amplitudes. QCD also predicts that the production cross section for charged $\rho$-pairs (with any helicity) is much larger that for that of neutral $\rho$ pairs, particularly at large $\theta_{\text {c.m. }}$ angles. Similar predictions are possible for other helicity-zero mesons. The cross sections for Compton scattering on protons and the crossed reaction $\gamma \gamma \rightarrow p \bar{p}$ at high momentum transfer have also been evaluated [97, 98], providing important tests of the proton distribution amplitude. 

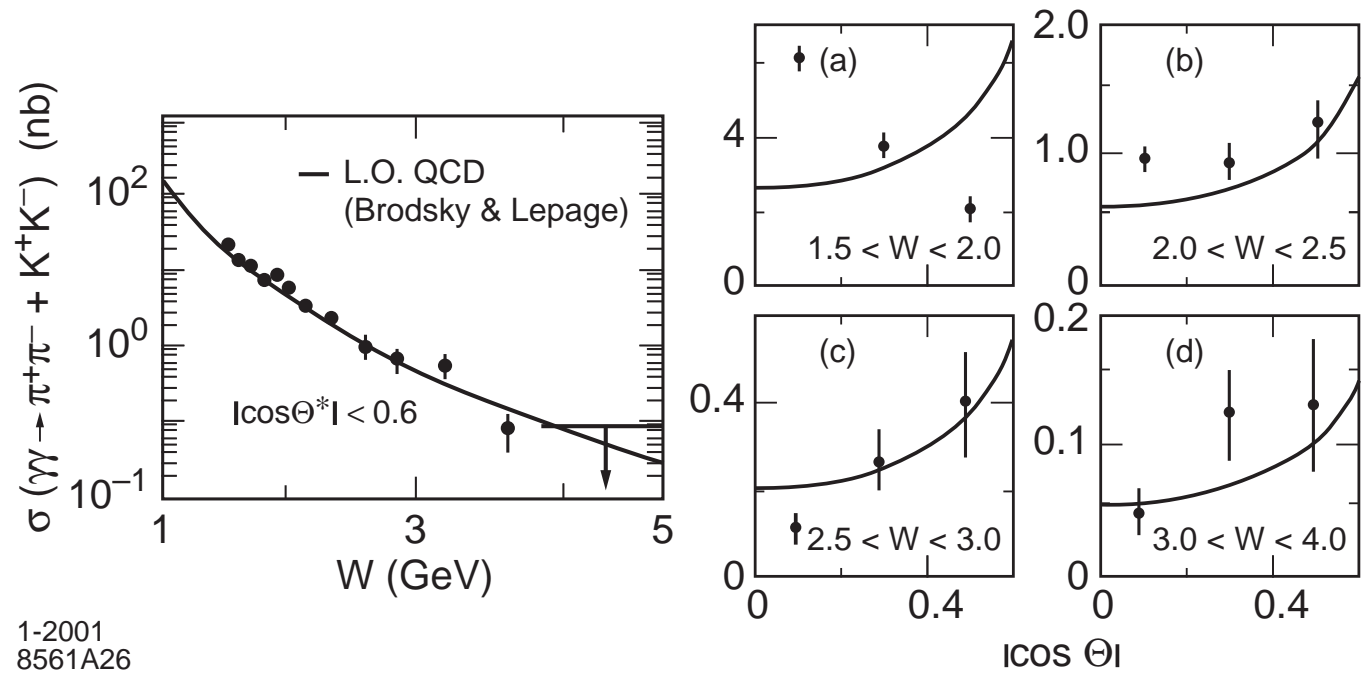

Figure 3: Comparison of the sum of $\gamma \gamma \rightarrow \pi^{+} \pi^{-}$and $\gamma \gamma \rightarrow K^{+} K^{-}$meson pair production cross sections with the scaling and angular distribution of the perturbative QCD prediction [90]. The data are from the CLEO collaboration [103].

It is particularly compelling to see a transition in angular dependence between the low energy chiral and PQCD regimes. The success of leading-twist perturbative QCD scaling for exclusive processes at presently experimentally accessible momentum transfer can be understood if the effective coupling $\alpha_{V}\left(Q^{*}\right)$ is approximately constant at the relatively small scales $Q^{*}$ relevant to the hard scattering amplitudes [89]. The evolution of the quark distribution amplitudes In the low- $Q^{*}$ domain at also needs to be minimal. Sudakov suppression of the endpoint contributions is also strengthened if the coupling is frozen because of the exponentiation of a double logarithmic series.

A debate has continued [99, 100, 101, 102] on whether processes such as the pion and proton form factors and elastic Compton scattering $\gamma p \rightarrow \gamma p$ might be dominated by higher-twist mechanisms until very large momentum transfer. If one assumes that the light-front wavefunction of the pion has the form $\psi_{\text {soft }}\left(x, k_{\perp}\right)=A \exp \left(-b \frac{k_{\perp}^{2}}{x(1-x)}\right)$, then the Feynman endpoint contribution to the overlap integral at small $k_{\perp}$ and $x \simeq 1$ will dominate the form factor compared to the hard-scattering contribution until very large $Q^{2}$. However, this ansatz for $\psi_{\text {soft }}\left(x, k_{\perp}\right)$ has no suppression at $k_{\perp}=0$ for any $x$; i.e., the wavefunction in the hadron rest frame does not fall-off at all for $k_{\perp}=0$ and $k_{z} \rightarrow-\infty$. Thus such wavefunctions do not represent well soft QCD contributions. Endpoint contributions are also suppressed by the QCD Sudakov form factor, reflecting the fact that a near-on-shell quark must radiate if it absorbs large momentum. One 
can show [5] that the leading power dependence of the two-particle light-front Fock wavefunction in the endpoint region is $1-x$, giving a meson structure function which falls as $(1-x)^{2}$ and thus by duality a non-leading contribution to the meson form factor $F\left(Q^{2}\right) \propto 1 / Q^{3}$. Thus the dominant contribution to meson form factors comes from the hard-scattering regime.

Radyushkin [100] has argued that the Compton amplitude is dominated by soft endpoint contributions of the proton wavefunctions where the two photons both interact on a quark line carrying nearly all of the proton's momentum. This description appears to agree with the Compton data at least at forward angles where $-t<10 \mathrm{GeV}^{2}$. From this viewpoint, the dominance of the factorizable PQCD leading twist contributions requires momentum transfers much higher than those currently available. However, the endpoint model cannot explain the empirical success of the perturbative QCD fixed $\theta_{c . m}$. scaling $s^{7} d \sigma / d t\left(\gamma p \rightarrow \pi^{+} n\right) \sim$ const at relatively low momentum transfer in pion photoproduction [104].

Clearly much more experimental input on hadron wavefunctions is needed, particularly from measurements of two-photon exclusive reactions into meson and baryon pairs at the high luminosity $B$ factories. For example, the ratio $\frac{d \sigma}{d t}\left(\gamma \gamma \rightarrow \pi^{0} \pi^{0}\right) / \frac{d \sigma}{d t}\left(\gamma \gamma \rightarrow \pi^{+} \pi^{-}\right)$ is particularly sensitive to the shape of pion distribution amplitude. Baryon pair production in two-photon reactions at threshold may reveal physics associated with the soliton structure of baryons in QCD [105, 106]. In addition, fixed target experiments can provide much more information on fundamental QCD processes such as deeply virtual Compton scattering and large angle Compton scattering.

\section{Self-Resolved Diffractive Reactions and Light Cone Wavefunctions}

Diffractive multi-jet production in heavy nuclei provides a novel way to measure the shape of the LC Fock state wavefunctions and test color transparency. For example, consider the reaction [2, 3, , 4] $\pi A \rightarrow \mathrm{Jet}_{1}+\mathrm{Jet}_{2}+A^{\prime}$ at high energy where the nucleus $A^{\prime}$ is left intact in its ground state. The transverse momenta of the jets balance so that

$\vec{k}_{\perp i}+\vec{k}_{\perp 2}=\vec{q}_{\perp}<R_{A}^{-1}$. The light-front longitudinal momentum fractions also need to add to $x_{1}+x_{2} \sim 1$ so that $\Delta p_{L}<R_{A}^{-1}$. The process can then occur coherently in the nucleus.

Because of color transparency, the valence wavefunction of the pion with small impact separation, will penetrate the nucleus with minimal interactions, diffracting 
into jet pairs [2]. The $x_{1}=x, x_{2}=1-x$ dependence of the di-jet distributions will thus reflect the shape of the pion valence light-front wavefunction in $x$; similarly, the $\vec{k}_{\perp 1}-\vec{k}_{\perp 2}$ relative transverse momenta of the jets gives key information on the derivative of the underlying shape of the valence pion wavefunction [3, ↔, 107]. The diffractive nuclear amplitude extrapolated to $t=0$ should be linear in nuclear number $A$ if color transparency is correct. The integrated diffractive rate should then scale as $A^{2} / R_{A}^{2} \sim A^{4 / 3}$. Preliminary results on a diffractive dissociation experiment of this type E791 at Fermilab using $500 \mathrm{GeV}$ incident pions on nuclear targets 108 appear to be consistent with color transparency. The measured longitudinal momentum distribution of the jets [109] is consistent with a pion light-cone wavefunction of the pion with the shape of the asymptotic distribution amplitude, $\phi_{\pi}^{\text {asympt }}(x)=\sqrt{3} f_{\pi} x(1-x)$. Data from CLEO 92 for the $\gamma \gamma^{*} \rightarrow \pi^{0}$ transition form factor also favor a form for the pion distribution amplitude close to the asymptotic solution to the perturbative QCD evolution equation [80, 5].

The diffractive dissociation of a hadron or nucleus can also occur via the Coulomb dissociation of a beam particle on an electron beam (e.g. at HERA or eRHIC) or on the strong Coulomb field of a heavy nucleus (e.g. at RHIC or nuclear collisions at the LHC) 107. The amplitude for Coulomb exchange at small momentum transfer is proportional to the first derivative $\sum_{i} e_{i} \frac{\partial}{\vec{k}_{T i}} \psi$ of the light-front wavefunction, summed over the charged constituents. The Coulomb exchange reactions fall off less fast at high transverse momentum compared to pomeron exchange reactions since the light-front wavefunction is effective differentiated twice in two-gluon exchange reactions.

It will also be interesting to study diffractive tri-jet production using proton beams $p A \rightarrow \mathrm{Jet}_{1}+\mathrm{Jet}_{2}+\mathrm{Jet}_{3}+A^{\prime}$ to determine the fundamental shape of the 3 -quark structure of the valence light-front wavefunction of the nucleon at small transverse separation [3]. For example, consider the Coulomb dissociation of a high energy proton at HERA. The proton can dissociate into three jets corresponding to the three-quark structure of the valence light-front wavefunction. We can demand that the produced hadrons all fall outside an opening angle $\theta$ in the proton's fragmentation region. Effectively all of the light-front momentum $\sum_{j} x_{j} \simeq 1$ of the proton's fragments will thus be produced outside an "exclusion cone". This then limits the invariant mass of the contributing Fock state $\mathcal{M}_{n}^{2}>\Lambda^{2}=P^{+2} \sin ^{2} \theta / 4$ from below, so that perturbative QCD counting rules can predict the fall-off in the jet system invariant mass $\mathcal{M}$. At large invariant mass one expects the three-quark valence Fock state of the proton to dominate. The segmentation of the forward detector in azimuthal angle $\phi$ can be used to identify structure and correlations associated with the three-quark light-front 
wavefunction [107]. An interesting possibility is that the distribution amplitude of the $\Delta(1232)$ for $J_{z}=1 / 2,3 / 2$ is close to the asymptotic form $x_{1} x_{2} x_{3}$, but that the proton distribution amplitude is more complex. This ansatz can also be motivated by assuming a quark-diquark structure of the baryon wavefunctions. The differences in shapes of the distribution amplitudes could explain why the $p \rightarrow \Delta$ transition form factor appears to fall faster at large $Q^{2}$ than the elastic $p \rightarrow p$ and the other $p \rightarrow N^{*}$ transition form factors [110. One can use also measure the dijet structure of real and virtual photons beams $\gamma^{*} A \rightarrow$ Jet $_{1}+$ Jet $_{2}+A^{\prime}$ to measure the shape of the light-front wavefunction for transversely-polarized and longitudinally-polarized virtual photons. Such experiments will open up a direct window on the amplitude structure of hadrons at short distances. The light-front formalism is also applicable to the description of nuclei in terms of their nucleonic and mesonic degrees of freedom [11], 112]. Self-resolving diffractive jet reactions in high energy electron-nucleus collisions and hadron-nucleus collisions at moderate momentum transfers can thus be used to resolve the light-front wavefunctions of nuclei.

\section{Higher Fock States and the Intrinsic Sea}

One can identify two contributions to the heavy quark sea, the "extrinsic" contributions which correspond to ordinary gluon splitting, and the "intrinsic" sea which is multiconnected via gluons to the valence quarks. The leading $1 / m_{Q}^{2}$ contributions to the intrinsic sea of the proton in the heavy quark expansion are proton matrix elements of the operator 113 $\eta^{\mu} \eta^{\nu} G_{\alpha \mu} G_{\beta \nu} G^{\alpha \beta}$ which in light-cone gauge $\eta^{\mu} A_{\mu}=A^{+}=0$ corresponds to three or four gluon exchange between the heavy-quark loop and the proton constituents in the forward virtual Compton amplitude. The intrinsic sea is thus sensitive to the hadronic bound-state structure [114, 35]. The maximal contribution of the intrinsic heavy quark occurs at $x_{Q} \simeq m_{\perp Q} / \sum_{i} m_{\perp}$ where $m_{\perp}=\sqrt{m^{2}+k_{\perp}^{2}}$; i.e. at large $x_{Q}$, since this minimizes the invariant mass $\mathcal{M}_{n}^{2}$. The measurements of the charm structure function by the EMC experiment are consistent with intrinsic charm at large $x$ in the nucleon with a probability of order $0.6 \pm 0.3 \%$ [36] which is consistent with the recent estimates based on instanton fluctuations [113].

Chang and Hou [115] have recently discussed the consequences of intrinsic charm in heavy quark states such as the $B, \Lambda_{B}$, and $\Upsilon$, such as an anomalous momentum distribution for $B \rightarrow J / \psi X$. The characteristic momenta characterizing the $B$ meson is most likely higher by a factor of 2 compared to the momentum scale of light mesons, This effect is analogous to the higher momentum scale of muonium $\mu^{+} e^{-}$versus that 
of positronium $e^{+} e^{-}$in atomic physics because of the larger reduced mass. Thus one can expect a higher probability for intrinsic charm in heavy hadrons compared to light hadrons.

One can also distinguish "intrinsic gluons" [116] which are associated with multiquark interactions and extrinsic gluon contributions associated with quark substructure. One can also use this framework to isolate the physics of the anomaly contribution to the Ellis-Jaffe sum rule [81]. Thus neither gluons nor sea quarks are solely generated by DGLAP evolution, and one cannot define a resolution scale $Q_{0}$ where the sea or gluon degrees of freedom can be neglected.

It is usually assumed that a heavy quarkonium state such as the $J / \psi$ always decays to light hadrons via the annihilation of its heavy quark constituents to gluons. However, as Karliner and I 117 have shown, the transition $J / \psi \rightarrow \rho \pi$ can also occur by the rearrangement of the $c \bar{c}$ from the $J / \psi$ into the $|q \bar{q} c \bar{c}\rangle$ intrinsic charm Fock state of the $\rho$ or $\pi$. On the other hand, the overlap rearrangement integral in the decay $\psi^{\prime} \rightarrow \rho \pi$ will be suppressed since the intrinsic charm Fock state radial wavefunction of the light hadrons will evidently not have nodes in its radial wavefunction. This observation provides a natural explanation of the long-standing puzzle [118 why the $J / \psi$ decays prominently to two-body pseudoscalar-vector final states, breaking hadron helicity conservation 88, whereas the $\psi^{\prime}$ does not.

The higher Fock state of the proton $|u u d s \bar{s}\rangle$ should resemble a $|K \Lambda\rangle$ intermediate state, since this minimizes its invariant mass $\mathcal{M}$. In such a state, the strange quark has a higher mean momentum fraction $x$ than the $\bar{s}$ [119, 8, 120]. Similarly, the helicity of the intrinsic strange quark in this configuration will be anti-aligned with the helicity of the nucleon [119, 120]. This $Q \leftrightarrow \bar{Q}$ asymmetry is a striking feature of the intrinsic heavy-quark sea.

\section{Non-Perturbative Solutions of Light-Front Quan- tized QCD}

Is there any hope of computing light-front wavefunctions from first principles? The

solution of the light-front Hamiltonian equation $H_{L C}^{Q C D}|\Psi\rangle=M^{2}|\Psi\rangle$ is an eigenvalue problem which in principle determines the masses squared of the entire bound and continuum spectrum of QCD. If one introduces periodic or anti-periodic boundary conditions, the eigenvalue problem is reduced to the diagonalization of a discrete Hermitian matrix representation of $H_{L C}^{Q C D}$. The light-front momenta satisfy $x^{+}=\frac{2 \pi}{L} n_{i}$ and 
$P^{+}=\frac{2 \pi}{L} K$, where $\sum_{i} n_{i}=K$. The number of quanta in the contributing Fock states is restricted by the choice of harmonic resolution. A cutoff on the invariant mass of the Fock states truncates the size of the matrix representation in the transverse momenta. This is the essence of the DLCQ method [121, which has now become a standard tool for solving both the spectrum and light-front wavefunctions of one-space one-time theories - virtually any $1+1$ quantum field theory, including "reduced QCD" (which has both quark and gluonic degrees of freedom) can be completely solved using DLCQ [122, 63. The method yields not only the bound-state and continuum spectrum, but also the light-front wavefunction for each eigensolution 123, 124.

In the case of theories in 3+1 dimensions, Hiller, McCartor, and I 125, 126] have recently shown that the use of covariant Pauli-Villars regularization with DLCQ allows one to obtain the spectrum and light-front wavefunctions of simplified theories, such as $(3+1)$ Yukawa theory. Dalley et al. have shown how one can use DLCQ in one spaceone time, with a transverse lattice to solve mesonic and gluonic states in $3+1$ QCD [127. The spectrum obtained for gluonium states is in remarkable agreement with lattice gauge theory results, but with a huge reduction of numerical effort. Hiller and I [128 have shown how one can use DLCQ to compute the electron magnetic moment in QED without resort to perturbation theory.

One can also formulate DLCQ so that supersymmetry is exactly preserved in the discrete approximation, thus combining the power of DLCQ with the beauty of supersymmetry [129, 130, 131]. The "SDLCQ" method has been applied to several interesting supersymmetric theories, to the analysis of zero modes, vacuum degeneracy, massless states, mass gaps, and theories in higher dimensions, and even tests of the Maldacena conjecture [129. Broken supersymmetry is interesting in DLCQ, since it may serve as a method for regulating non-Abelian theories [126].

There are also many possibilities for obtaining approximate solutions of light-front wavefunctions in QCD. QCD sum rules, lattice gauge theory moments, and QCD inspired models such as the bag model, chiral theories, provide important constraints. Guides to the exact behavior of LC wavefunctions in QCD can also be obtained from analytic or DLCQ solutions to toy models such as "reduced" $Q C D(1+1)$. The lightfront and many-body Schrödinger theory formalisms must match In the nonrelativistic limit.

It would be interesting to see if light-front wavefunctions can incorporate chiral constraints such as soliton (Skyrmion) behavior for baryons and other consequences of the chiral limit in the soft momentum regime. Solvable theories such as $Q C D(1+1)$ are also useful for understanding such phenomena. It has been shown that the anomaly 
contribution for the $\pi^{0} \rightarrow \gamma \gamma$ decay amplitude is satisfied by the light-front Fock formalism in the limit where the mass of the pion is light compared to its size 132.

\section{Non-Perturbative Calculations of the Pion Dis- tribution Amplitude}

The distribution amplitude $\phi(x, \widetilde{Q})$ can be computed from the integral over transverse momenta of the renormalized hadron valence wavefunction in the light-cone gauge at fixed light-cone time [16]:

$$
\phi(x, \widetilde{Q})=\int d^{2} \overrightarrow{k_{\perp}} \theta\left(\widetilde{Q}^{2}-\frac{\vec{k}_{\perp}^{2}}{x(1-x)}\right) \psi^{(\widetilde{Q})}\left(x, \overrightarrow{k_{\perp}}\right)
$$

where a global cutoff in invariant mass is identified with the resolution $\tilde{Q}$. The distribution amplitude $\phi(x, \tilde{Q})$ is boost and gauge invariant and evolves in $\ln \tilde{Q}$ through an evolution equation [83, 80, 5]. Since it is formed from the same product of operators as the non-singlet structure function, the anomalous dimensions controlling $\phi(x, Q)$ dependence in the ultraviolet $\log Q$ scale are the same as those which appear in the DGLAP evolution of structure functions 455. The decay $\pi \rightarrow \mu \nu$ normalizes the wave function at the origin: $a_{0} / 6=\int_{0}^{1} d x \phi(x, Q)=f_{\pi} /(2 \sqrt{3})$. One can also compute the distribution amplitude from the gauge invariant Bethe-Salpeter wavefunction at equal light-cone time. This also allows contact with both QCD sum rules and lattice gauge theory; for example, moments of the pion distribution amplitudes have been computed in lattice gauge theory [9, 133, 134.

Dalley 135 has recently calculated the pion distribution amplitude from QCD using a combination of the discretized DLCQ method for the $x^{-}$and $x^{+}$light-cone coordinates with the transverse lattice method [136, 137] in the transverse directions, A finite lattice spacing $a$ can be used by choosing the parameters of the effective theory in a region of renormalization group stability to respect the required gauge, Poincaré, chiral, and continuum symmetries. The overall normalization gives $f_{\pi}=101 \mathrm{MeV}$ compared with the experimental value of $93 \mathrm{MeV}$. Figure 6 (a) compares the resulting DLCQ/transverse lattice pion wavefunction with the best fit to the diffractive di-jet data (see the next section) after corrections for hadronization and experimental acceptance [1]. The theoretical curve is somewhat broader than the experimental result. However, there are experimental uncertainties from hadronization and theoretical errors introduced from finite DLCQ resolution, using a nearly massless pion, ambiguities 
in setting the factorization scale $Q^{2}$, as well as errors in the evolution of the distribution amplitude from 1 to $10 \mathrm{GeV}^{2}$. Instanton models also predict a pion distribution amplitude close to the asymptotic form [138. In contrast, recent lattice results from Del Debbio et al. 134 predict a much narrower shape for the pion distribution amplitude than the distribution predicted by the transverse lattice. A new result for the proton distribution amplitude treating nucleons as chiral solitons has recently been derived by Diakonov and Petrov [139]. Dyson-Schwinger models [10] of hadronic Bethe-Salpeter wavefunctions can also be used to predict light-cone wavefunctions and hadron distribution amplitudes by integrating over the relative $k^{-}$momentum. There is also the possibility of deriving Bethe-Salpeter wavefunctions within light-cone gauge quantized QCD [21] in order to properly match to the light-cone gauge Fock state decomposition.
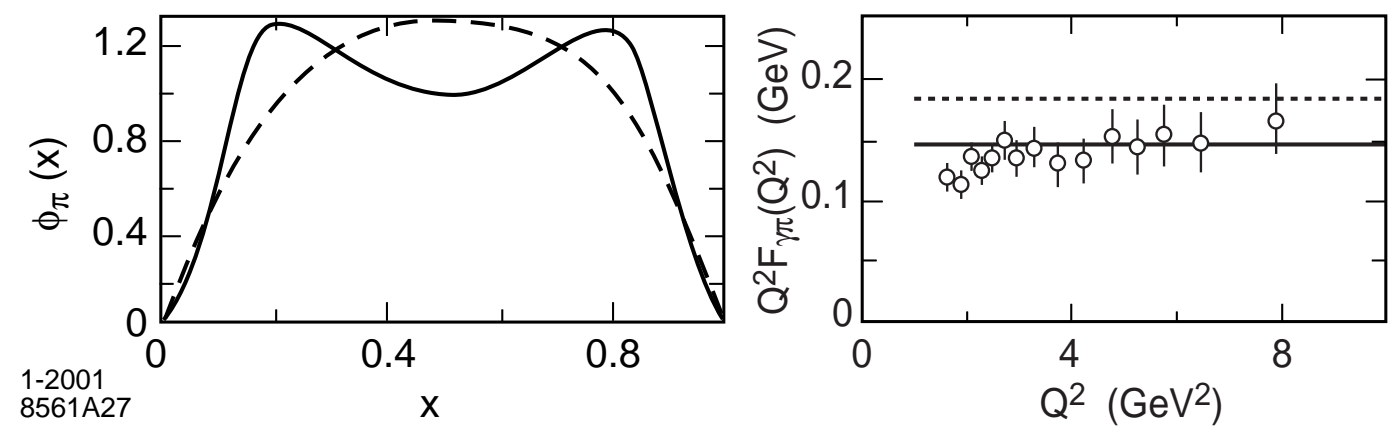

Figure 4: (a) Preliminary transverse lattice results for the pion distribution amplitude at $Q^{2} \sim 10 \mathrm{GeV}^{2}$. The solid curve is the theoretical prediction from the combined DLCQ/transverse lattice method [135]; the chain line is the experimental result obtained from jet diffractive dissociation [1]. Both are normalized to the same area for comparison. (b) Scaling of the transition photon to pion transition form factor $Q^{2} F_{\gamma \pi^{0}}\left(Q^{2}\right)$. The dotted and solid theoretical curves are the perturbative QCD prediction at leading and next-to-leading order, respectively, assuming the asymptotic pion distribution The data are from the CLEO collaboration [92].

\section{Conclusions}

In these lectures I have shown how the universal, process-independent and frameindependent light-front Fock-state wavefunctions encode the properties of a hadron in terms of its fundamental quark and gluon degrees of freedom. Knowledge of such wavefunctions will be critical for progress in understanding exclusive $B$ decays. 
I have shown how, given the proton's light-front wavefunctions, one can compute not only the moments of the quark and gluon distributions measured in deep inelastic lepton-proton scattering, but also the multi-parton correlations which control the distribution of particles in the proton fragmentation region and dynamical higher twist effects. Light-front wavefunctions also provide a systematic framework for evaluating exclusive hadronic matrix elements, including time-like heavy hadron decay amplitudes, form factors, and the generalized form factors that appear in deeply virtual Compton scattering. The light-front Hamiltonian formalism also provides a physical factorization scheme for separating hard and soft contributions in both exclusive and inclusive hard processes.

The leading-twist QCD predictions for exclusive two-photon processes such as the photon-to-pion transition form factor and $\gamma \gamma \rightarrow$ hadron pairs are based on rigorous factorization theorems. The recent data from the CLEO collaboration on $F_{\gamma \pi}\left(Q^{2}\right)$ and the sum of $\gamma \gamma \rightarrow \pi^{+} \pi^{-}$and $\gamma \gamma \rightarrow K^{+} K^{-}$channels are in excellent agreement with the QCD predictions. It is particularly compelling to see a transition in angular dependence between the low energy chiral and PQCD regimes. The success of leading-twist perturbative QCD scaling for exclusive processes at presently experimentally accessible momentum transfer can be understood if the effective coupling $\alpha_{V}\left(Q^{*}\right)$ is approximately constant at the relatively small scales $Q^{*}$ relevant to the hard scattering amplitudes [89]. The evolution of the quark distribution amplitudes In the low- $Q^{*}$ domain at also needs to be minimal. Sudakov suppression of the endpoint contributions is also strengthened if the coupling is frozen because of the exponentiation of a double logarithmic series.

One of the formidable challenges in QCD is the calculation of non-perturbative wavefunctions of hadrons from first principles. The recent calculation of the pion distribution amplitude by Dalley [135] using light-cone and transverse lattice methods is particularly encouraging. The predicted form of $\phi_{\pi}(x, Q)$ is somewhat broader than but not inconsistent with the asymptotic form favored by the measured normalization of $Q^{2} F_{\gamma \pi^{0}}\left(Q^{2}\right)$ and the pion wavefunction inferred from diffractive di-jet production.

Clearly much more experimental input on hadron wavefunctions is needed, particularly from measurements of two-photon exclusive reactions into meson and baryon pairs at the high luminosity $B$ factories. For example, the ratio $\frac{d \sigma}{d t}\left(\gamma \gamma \rightarrow \pi^{0} \pi^{0}\right) / \frac{d \sigma}{d t}\left(\gamma \gamma \rightarrow \pi^{+} \pi^{-}\right)$ is particularly sensitive to the shape of pion distribution amplitude. Baryon pair production in two-photon reactions at threshold may reveal physics associated with the soliton structure of baryons in QCD [105. In addition, fixed target experiments can provide much more information on fundamental QCD processes such as deeply virtual Compton scattering and large angle Compton scattering. 
A remarkable new type of diffractive jet production reaction, "self-resolving diffractive interactions" can provide direct empirical information on the light-front wavefunctions of hadrons. The recent E791 experiment at Fermilab has not only determined the main features of the pion wavefunction, but has also confirmed color transparency, a fundamental test of the gauge properties of QCD. Analogous reaction involving nuclear projectiles can resolve the light-front wavefunctions of nuclei in terms of their nucleon and mesonic degrees of freedom. It is also possible to measure the light-front wavefunctions of atoms through high energy Coulomb dissociation.

There has been notable progress in computing light-front wavefunctions directly from the QCD light-front Hamiltonian, using DLCQ and transverse lattice methods. Even without full non-perturbative solutions of QCD, one can envision a program to construct the light-front wavefunctions using measured moments constraints from QCD sum rules, lattice gauge theory, and data from hard exclusive and inclusive processes. One can also be guided by theoretical constraints from perturbation theory which dictate the asymptotic form of the wavefunctions at large invariant mass, $x \rightarrow 1$, and high $k_{\perp}$. One can also use ladder relations which connect Fock states of different particle number; perturbatively-motivated numerator spin structures; conformal symmetry,

guidance from toy models such as "reduced" $Q C D(1+1)$; and the correspondence to Abelian theory for $N_{C} \rightarrow 0$, as well as many-body Schrödinger theory in the nonrelativistic domain.

\section{Acknowledgments}

Work supported by the Department of Energy under contract number DE-AC0376SF00515. I wish to thank the organizers of this meeting, Hsiang-nan Li and Wei-Min Zhang, for their outstanding hospitality in Taiwan. Much of this work is based on collaborations, particularly with Markus Diehl, Paul Hoyer, Dae Sung Hwang, Peter Lepage, Bo-Qiang Ma, Hans Christian Pauli, Johan Rathsman, Ivan Schmidt, and Prem Srivastava.

\section{References}

[1] D. Ashery [E791 Collaboration], hep-ex/9910024.

[2] G. Bertsch, S. J. Brodsky, A. S. Goldhaber and J. F. Gunion, Phys. Rev. Lett. 47, 297 (1981). 
[3] L. Frankfurt, G. A. Miller and M. Strikman, Phys. Lett. B304, 1 (1993) hepph/9305228.

[4] L. Frankfurt, G. A. Miller and M. Strikman, Found. Phys. 30, 533 (2000) hepph/9907214.

[5] G. P. Lepage and S. J. Brodsky, Phys. Rev. D 22, 2157 (1980).

[6] M. Beneke, G. Buchalla, M. Neubert and C. T. Sachrajda, Phys. Rev. Lett. 83, 1914 (1999) hep-ph/9905312.

[7] Y. Keum, H. Li and A. I. Sanda, hep-ph/0004004.

[8] A. I. Signal and A. W. Thomas, Phys. Lett. B191, 205 (1987).

[9] G. Martinelli and C. T. Sachrajda, Amplitude," Phys. Lett. B190, 151 (1987).

[10] M. B. Hecht, C. D. Roberts and S. M. Schmidt, nucl-th/0008049.

[11] D. G. Robertson, E. S. Swanson, A. P. Szczepaniak, C. R. Ji and S. R. Cotanch, Phys. Rev. D 59, 074019 (1999) hep-ph/9811224.

[12] S. J. Brodsky, H. Pauli and S. S. Pinsky, Phys. Rept. 301, 299 (1998) hep$\mathrm{ph} / 9705477$.

[13] P. A. Dirac, Rev. Mod. Phys. 21, 392 (1949).

[14] S. Weinberg, Phys. Rev. 150, 1313 (1966).

[15] S. J. Brodsky, R. Roskies and R. Suaya, Momentum Frame," Phys. Rev. D 8, 4574 (1973).

[16] S. J. Brodsky and G. P. Lepage, in Perturbative Quantum Chromodynamics, A. H. Mueller, Ed. (World Scientific, 1989).

[17] S. J. Brodsky, D. S. Hwang, B. Ma and I. Schmidt, Nucl. Phys. B593, 311 (2001) hep-th/0003082.

[18] P. P. Srivastava and S. J. Brodsky, hep-ph/0011372.

[19] J. M. Cornwall and J. Papavassiliou, Phys. Rev. D 40, 3474 (1989).

[20] S. J. Brodsky, E. Gardi, G. Grunberg and J. Rathsman, hep-ph/0002065. 
[21] P. P. Srivastava and S. J. Brodsky, Phys. Rev. D61, 025013 (2000), hep-ph/9906423, and SLAC-PUB 8543, in preparation.

[22] A. Bassetto, L. Griguolo and F. Vian, hep-th/9911036.

[23] K. Yamawaki, hep-th/9802037.

[24] G. McCartor, hep-th/0004139.

[25] P. P. Srivastava, Phys. Lett. B448, 68 (1999) hep-th/9811225.

[26] S. S. Pinsky and B. van de Sande, Phys. Rev. D49, 2001 (1994), hep-ph/9310330.

[27] S. D. Drell and T. Yan, Phys. Rev. Lett. 24, 181 (1970).

[28] G. B. West, Phys. Rev. Lett. 24, 1206 (1970).

[29] S. J. Brodsky and S. D. Drell, Phys. Rev. D 22, 2236 (1980).

[30] S. J. Brodsky and D. S. Hwang, Nucl. Phys. B543, 239 (1999) hep-ph/9806358.

[31] S. J. Brodsky, M. Diehl and D. S. Hwang, hep-ph/0009254.

[32] M. Diehl, T. Feldmann, R. Jakob and P. Kroll, hep-ph/0009255.

[33] S. J. Brodsky, M. Burkardt and I. Schmidt, distributions," Nucl. Phys. B441, 197 (1995) hep-ph/9401328.

[34] S. J. Brodsky, hep-ph/0006310.

[35] S. J. Brodsky, P. Hoyer, C. Peterson and N. Sakai, Phys. Lett. B93, 451 (1980).

[36] B. W. Harris, J. Smith and R. Vogt, Nucl. Phys. B461, 181 (1996) hep$\mathrm{ph} / 9508403$.

[37] F. Antonuccio, S. J. Brodsky and S. Dalley, Phys. Lett. B412, 104 (1997) hepph/9705413.

[38] S. J. Brodsky and A. H. Mueller, Phys. Lett. B206, 685 (1988).

[39] L. L. Frankfurt and M. I. Strikman, Phys. Rept. 160, 235 (1988).

[40] S. J. Brodsky and B. T. Chertok, Phys. Rev. D 14, 3003 (1976).

[41] S. J. Brodsky, C. Ji and G. P. Lepage, Phys. Rev. Lett. 51, 83 (1983). 
[42] G. R. Farrar, K. Huleihel and H. Zhang, Phys. Rev. Lett. 74, 650 (1995).

[43] S. J. Brodsky, E. Chudakov, P. Hoyer and J. M. Laget, hep-ph/0010343.

[44] S. J. Brodsky and J. Rathsman, hep-ph/9906339.

[45] S. J. Brodsky, Y. Frishman, G. P. Lepage and C. Sachrajda, Phys. Lett. 91B, 239 (1980).

[46] D. Müller, Phys. Rev. D49, 2525 (1994).

[47] V. M. Braun, S. E. Derkachov, G. P. Korchemsky and A. N. Manashov, Nucl. Phys. B553, 355 (1999), hep-ph/9902375.

[48] S. J. Brodsky and H. J. Lu, Phys. Rev. D51, 3652 (1995), hep-ph/9405218.

[49] S. J. Brodsky, J. R. Pelaez and N. Toumbas, Phys. Rev. D60, 037501 (1999), hep-ph/9810424.

[50] S. J. Brodsky, G. T. Gabadadze, A. L. Kataev and H. J. Lu, Phys. Lett. B372, 133 (1996) hep-ph/9512367.

[51] S. J. Brodsky, M. S. Gill, M. Melles and J. Rathsman, Phys. Rev. D 58, 116006 (1998) hep-ph/9801330.

[52] S. J. Brodsky and P. Huet, Phys. Lett. B417, 145 (1998), hep-ph/9707543.

[53] L. Okun and I. Yu. Kobzarev, ZhETF, 431904 (1962) ( English translation : JETP 161343 (1963)); L. Okun, in proceedings of the International Conference on Elementary Particles, 4th, Heidelberg, Germany (1967). Edited by H. Filthuth. North-Holland, (1968).

[54] X. Ji, hep-ph/9610369.

[55] X. Ji, Phys. Rev. Lett. 78, 610 (1997), hep-ph/9603249.

[56] X. Ji, Phys. Rev. D55, 7114 (1997), hep-ph/9609381.

[57] O. V. Teryaev, hep-ph/9904376.

[58] A. Harindranath and R. Kundu, Phys. Rev. D59, 116013 (1999), hep-ph/9802406.

[59] S. Chang, R. G. Root and T. Yan, Phys. Rev. D 7, 1133 (1973). 
[60] M. Burkardt, Nucl. Phys. A504, 762 (1989).

[61] H.-M. Choi and C.-R. Ji, Phys. Rev. D 58, 071901 (1998).

[62] K. Hornbostel, S. J. Brodsky and H. C. Pauli, Phys. Rev. D 41, 3814 (1990).

[63] F. Antonuccio and S. Dalley, Phys. Lett. B348, 55 (1995) hep-th/9411204.

[64] S. J. Brodsky, F. E. Close and J. F. Gunion, Phys. Rev. D 5, 1384 (1972).

[65] S. J. Brodsky, F. E. Close and J. F. Gunion, Phys. Rev. D 6, 177 (1972).

[66] S. J. Brodsky, F. E. Close and J. F. Gunion, Phys. Rev. D 8, 3678 (1973).

[67] M. Diehl, T. Gousset and B. Pire, Phys. Rev. D 62, 073014 (2000) hep$\mathrm{ph} / 0003233$.

[68] A. V. Radyushkin, Phys. Lett. B380, 417 (1996) hep-ph/9604317.

[69] X. Ji and J. Osborne, Phys. Rev. D 58, 094018 (1998) hep-ph/9801260.

[70] P. A. Guichon and M. Vanderhaeghen, Prog. Part. Nucl. Phys. 41, 125 (1998) hep-ph/9806305.

[71] M. Vanderhaeghen, P. A. Guichon and M. Guidal, Phys. Rev. Lett. 80, 5064 (1998).

[72] A. V. Radyushkin, Phys. Rev. D 59, 014030 (1999) hep-ph/9805342.

[73] J. C. Collins and A. Freund, Phys. Rev. D 59, 074009 (1999) hep-ph/9801262.

[74] M. Diehl, T. Feldmann, R. Jakob and P. Kroll, Phys. Lett. B460, 204 (1999) hep-ph/9903268.

[75] M. Diehl, T. Feldmann, R. Jakob and P. Kroll, Eur. Phys. J. C8, 409 (1999) hep-ph/9811253.

[76] J. Blumlein and D. Robaschik, Nucl. Phys. B581, 449 (2000) hep-ph/0002071.

[77] M. Penttinen, M. V. Polyakov, A. G. Shuvaev and M. Strikman, Phys. Lett. B491, 96 (2000) hep-ph/0006321.

[78] D. Muller, D. Robaschik, B. Geyer, F. M. Dittes and J. Horejsi, Fortsch. Phys. 42, 101 (1994) hep-ph/9812448. 
[79] S. J. Brodsky, SLAC-PUB-8649.

[80] G. P. Lepage and S. J. Brodsky, Phys. Lett. B 87, 359 (1979).

[81] S. D. Bass, S. J. Brodsky and I. Schmidt, Phys. Rev. D 60, 034010 (1999) hepph/9901244.

[82] S. J. Brodsky and G. R. Farrar, Phys. Rev. D 11, 1309 (1975).

[83] G. P. Lepage and S. J. Brodsky, Phys. Rev. Lett. 43, 545 (1979).

[84] A. Szczepaniak, E. M. Henley and S. J. Brodsky, Phys. Lett. B243, 287 (1990).

[85] A. Szczepaniak, Phys. Rev. D 54, 1167 (1996).

[86] Y. Y. Keum, H. Li and A. I. Sanda, hep-ph/0004173.

[87] H. Li, hep-ph/0012140.

[88] S. J. Brodsky and G. P. Lepage, Phys. Rev. D 24, 2848 (1981).

[89] S. J. Brodsky, C. Ji, A. Pang and D. G. Robertson, Phys. Rev. D 57, 245 (1998) hep-ph/9705221.

[90] S. J. Brodsky and G. P. Lepage, in C81-04-06.1.4 Phys. Rev. D 24, 1808 (1981).

[91] E. Braaten and S. Tse, Phys. Rev. D 35, 2255 (1987).

[92] J. Gronberg et al. [CLEO Collaboration], Phys. Rev. D57, 33 (1998), hep-ex/9707031; and H. Paar, presented at PHOTON 2000: International Workshop on Structure and Interactions of the Photon Ambleside, Lake District, England, 26-31 Aug 2000.

[93] A. V. Radyushkin, Acta Phys. Polon. B26, 2067 (1995) hep-ph/9511272].

[94] S. Ong, Phys. Rev. D 52, 3111 (1995).

[95] P. Kroll and M. Raulfs, Phys. Lett. B387, 848 (1996) hep-ph/9605264.

[96] V. L. Chernyak and A. R. Zhitnitsky, Phys. Rept. 112, 173 (1984).

[97] G. R. Farrar and H. Zhang, Phys. Rev. Lett. 65, 1721 (1990).

[98] T. C. Brooks and L. Dixon, Phys. Rev. D 62, 114021 (2000) hep-ph/0004143. 
[99] N. Isgur and C. H. Llewellyn Smith, Phys. Lett. B217, 535 (1989).

[100] A. V. Radyushkin, Phys. Rev. D 58, 114008 (1998) hep-ph/9803316.

[101] J. Bolz and P. Kroll, Z. Phys. A356, 327 (1996) [hep-ph/9603289.

[102] C. Vogt, hep-ph/0010040.

[103] Paar, H., et al. CLEO collaboration (to be published); See also Boyer, J. et al., Phys. Rev. Lett. 56, 207 (1980); TPC/Two Gamma Collaboration (H. Aihara et al.), Phys. Rev. Lett. 57,404 (1986).

[104] R. L. Anderson et al., Phys. Rev. Lett. 30, 627 (1973).

[105] H. M. Sommermann, R. Seki, S. Larson and S. E. Koonin, Phys. Rev. D 45, 4303 (1992).

[106] S. Brodsky and M. Karliner, in preparation.

[107] S. Brodsky, M. Diehl, P. Hoyer, and S. Peigne, in preparation.

[108] E. M. Aitala et al. [E791 Collaboration], hep-ex/0010044.

[109] E. M. Aitala et al. [E791 Collaboration], pion light-cone wave function squared," hep-ex/0010043.

[110] P. Stoler, Few Body Syst. Suppl. 11, 124 (1999).

[111] G. A. Miller, nucl-th/9910053.

[112] G. A. Miller, S. J. Brodsky and M. Karliner, Phys. Lett. B481, 245 (2000) hep$\mathrm{ph} / 0002156$.

[113] M. Franz, M. V. Polyakov and K. Goeke, Phys. Rev. D 62, 074024 (2000) hep$\mathrm{ph} / 0002240$.

[114] S. J. Brodsky, C. Peterson and N. Sakai, Phys. Rev. D 23, 2745 (1981).

[115] C. V. Chang and W. Hou, in B Meson," hep-ph/0101162.

[116] S. J. Brodsky and I. A. Schmidt, Phys. Lett. B234, 144 (1990).

[117] S. J. Brodsky and M. Karliner, Phys. Rev. Lett. 78, 4682 (1997) hepph/9704379. 
[118] S. J. Brodsky, G. P. Lepage and S. F. Tuan, Phys. Rev. Lett. 59, 621 (1987).

[119] M. Burkardt and B. Warr, Phys. Rev. D 45, 958 (1992).

[120] S. J. Brodsky and B. Ma, Phys. Lett. B381, 317 (1996) hep-ph/9604393.

[121] H. C. Pauli and S. J. Brodsky, Phys. Rev. D 32, 2001 (1985).

[122] S. Dalley and I. R. Klebanov, Phys. Rev. D 47, 2517 (1993) hep-th/9209049.

[123] F. Antonuccio and S. Dalley, Phys. Lett. B376, 154 (1996) hep-th/9512106.

[124] F. Antonuccio and S. Dalley, Nucl. Phys. B461, 275 (1996) hep-ph/9506456.

[125] S. J. Brodsky, J. R. Hiller and G. McCartor, Phys. Rev. D 58, 025005 (1998) hep-th/9802120.

[126] S. J. Brodsky, J. R. Hiller and G. McCartor, Phys. Rev. D 60, 054506 (1999) hep-ph/9903388.

[127] S. Dalley and B. van de Sande, Phys. Rev. D 62, 014507 (2000) hep-lat/9911035.

[128] J. R. Hiller and S. J. Brodsky, Phys. Rev. D 59, 016006 (1999) hep-ph/9806541.

[129] F. Antonuccio, I. Filippov, P. Haney, O. Lunin, S. Pinsky, U. Trittmann and J. Hiller [SDLCQ Collaboration], hep-th/9910012. Brodsky:1999xj

[130] O. Lunin and S. Pinsky, hep-th/9910222.

[131] P. Haney, J. R. Hiller, O. Lunin, S. Pinsky and U. Trittmann, Phys. Rev. D 62, 075002 (2000) [hep-th/9911243].

[132] G. P. Lepage, S. J. Brodsky, T. Huang and P. B. Mackenzie, CLNS-82/522, published in Banff Summer Inst.1981:0083 (QCD161:B23:1981); S. J. Brodsky, T. Huang and G. P. Lepage, In *Banff 1981, Proceedings, Particles and Fields 2*, $143-199$.

[133] D. Daniel, R. Gupta and D. G. Richards, Phys. Rev. D 43, 3715 (1991).

[134] L. Del Debbio, M. Di Pierro, A. Dougall and C. Sachrajda [UKQCD collaboration], Nucl. Phys. Proc. Suppl. 83, 235 (2000) hep-lat/9909147].

[135] S. Dalley, hep-ph/0007081. 
[136] W. A. Bardeen and R. B. Pearson, Phys. Rev. D 14, 547 (1976).

[137] M. Burkardt, Phys. Rev. D 54, 2913 (1996) hep-ph/9601289.

[138] V. Y. Petrov, M. V. Polyakov, R. Ruskov, C. Weiss and K. Goeke, Phys. Rev. D 59, 114018 (1999) [hep-ph/9807229].

[139] D. Diakonov and V. Y. Petrov, hep-ph/0009006. 